\title{
SHARPIN-mediated regulation of protein arginine methyltransferase 5 controls melanoma growth
}

\author{
Hironari Tamiya, ${ }^{1}$ Hyungsoo Kim, ${ }^{1}$ Oleksiy Klymenko, ${ }^{2}$ Heejung Kim, ${ }^{1}$ Yongmei Feng, ${ }^{1}$ Tongwu Zhang, ${ }^{3}$ Jee Yun Han, ${ }^{1}$ \\ Ayako Murao, ${ }^{1}$ Scott J. Snipas, ${ }^{1}$ Lucia Jilaveanu, ${ }^{4}$ Kevin Brown, ${ }^{3}$ Harriet Kluger, ${ }^{4}$ Hao Zhang, ${ }^{5}$ Kazuhiro Iwai, ${ }^{6}$ and Ze'ev A. Ronai ${ }^{1,2}$ \\ ${ }^{1}$ Tumor Initiation and Maintenance Program, Cancer Center, Sanford Burnham Prebys Medical Discovery Institute, La Jolla, California, USA. ${ }^{2}$ Technion Integrated Cancer Center, Technion Israel Institute of \\ Technology, Haifa, Israel. ${ }^{3}$ Division of Cancer Epidemiology and Genetics, Laboratory of Translational Genomics, National Cancer Institute, Bethesda, Maryland, USA. ${ }^{4}$ Department of Internal Medicine, \\ Section of Medical Oncology, Yale University, New Haven, Connecticut, USA. ${ }^{5}$ Cancer Research Center, Shantou University Medical College, Shantou, Guangdong, China. ${ }^{6}$ Department of Molecular and Cellular \\ Physiology, Graduate School of Medicine, Kyoto University, Kyoto, Japan.
}

\begin{abstract}
SHARPIN, an adaptor for the linear ubiquitin chain assembly complex (LUBAC), plays important roles in NF- $\kappa B$ signaling and inflammation. Here, we have demonstrated a LUBAC-independent role for SHARPIN in regulating melanoma growth. We observed that SHARPIN interacted with PRMT5, a type II protein arginine methyltransferase, and increased its multiprotein complex and methyltransferase activity. Activated PRMT5 controlled the expression of the transcription factors SOX10 and MITF by SHARPIN-dependent arginine dimethylation and inhibition of the transcriptional corepressor SKI. Activation of PRMT5 by SHARPIN counteracted PRMT5 inhibition by methylthioadenosine, a substrate of methylthioadenosine phosphorylase, which is codeleted with cyclin-dependent kinase inhibitor 2A (CDKN2A) in approximately $15 \%$ of human cancers. Collectively, we identified a LUBAC-independent role for SHARPIN in enhancing PRMT5 activity that contributes to melanomagenesis through the SKI/SOX10 regulatory axis.
\end{abstract}

\section{Introduction}

Concerted regulation of posttranslational protein modification is central to the maintenance of cellular homeostasis, and not surprisingly, such modification is often deregulated in pathophysiological conditions, including cancer. Ubiquitination and methylation are two of the many posttranslational modifications that define the spatial and temporal regulation of cellular signaling. Although we have a good mechanistic understanding of ubiquitination and methylation as individual modifications, the extent to which they may control protein function by acting in concert is poorly understood.

The linear ubiquitin chain assembly complex (LUBAC) is composed of 3 structurally related proteins: HOIL-1 interacting protein/ RNF31 (HOIP), heme-oxidized IRP2 ligase 1L/RBCK1 (HOIL-1L), and SHANK-associated $\mathrm{RH}$ domain-interacting protein (SHARPIN). LUBAC forms linear-type ubiquitin chains through methionine 1-glycine 76 linkages (1-3). Linear ubiquitination has been implicated in the regulation of canonical NF- $\mathrm{KB}$ signaling; accordingly, defects in this process are associated with inflammatory immune disorders and cancer $(4,5)$. Similarly, altered expression of HOIP or OTULIN, a linear ubiquitin chain-specific deubiquitinase, plays a role in autoimmunity and lymphomagenesis $(4,6)$. Intriguingly, SHARPIN is known to affect the activity of key signaling pathways, as shown for glioma and prostate cancer cells (7). Given the high

Authorship note: H. Tamiya and H. Kim contributed equally to this work. Conflict of interest: The authors have declared that no conflict of interest exists. Submitted: May 31, 2017; Accepted: October 31, 2017.

Reference information: J Clin Invest. 2018;128(1):517-530.

https://doi.org/10.1172/JCI95410 expression of SHARPIN in prostate, breast, and lung cancer, this finding points to a potential LUBAC-independent role for SHARPIN in the growth and progression of certain cancer types $(8,9)$.

We identified PRMT5, a type II protein arginine methyltransferase, as a SHARPIN-binding protein. PRMT5 catalyzes the monomethylation and symmetric dimethylation of arginine (SDME-RG) in diverse substrates such as histones and nonhistone proteins involved in transcription, splicing, and translation as well as other cellular processes. PRMT5 was previously shown to affect TP53, the eukaryotic translation initiation factor eIF4E, the androgen receptor, and the epidermal growth factor receptor, pointing to a potentially important role in cancer biology (10-12). PRMT5 is inhibited by 5'-O-methylthioadenosine (MTA), the substrate of methylthioadenosine phosphorylase (MTAP), which is a key enzyme in the methionine salvage pathway. Notably, MTAP is codeleted with cyclin-dependent kinase inhibitor $2 \mathrm{~A}(C D K N 2 A)$ in approximately $15 \%$ of human tumors, resulting in MTA accumulation and suppression of PRMT5 activity. As a result, $M T A P / C D$ KN2A-deleted tumors are sensitive to agents that inhibit residual PRMT5 activity (13-15).

Here, we investigated the significance and the implications of SHARPIN-PRMT5 binding for human melanoma. Our findings strongly suggest a role for SHARPIN-PRMT5 in the transcriptional regulation of $S O X 10, P A X 3$, and $M I T F, 3$ transcription factors that play pivotal roles in melanomagenesis.

\section{Results}

SHARPIN expression and importance in melanoma. We examined the expression of LUBAC components in multiple cancer types and found that SHARPIN was upregulated in many cancers, 
A
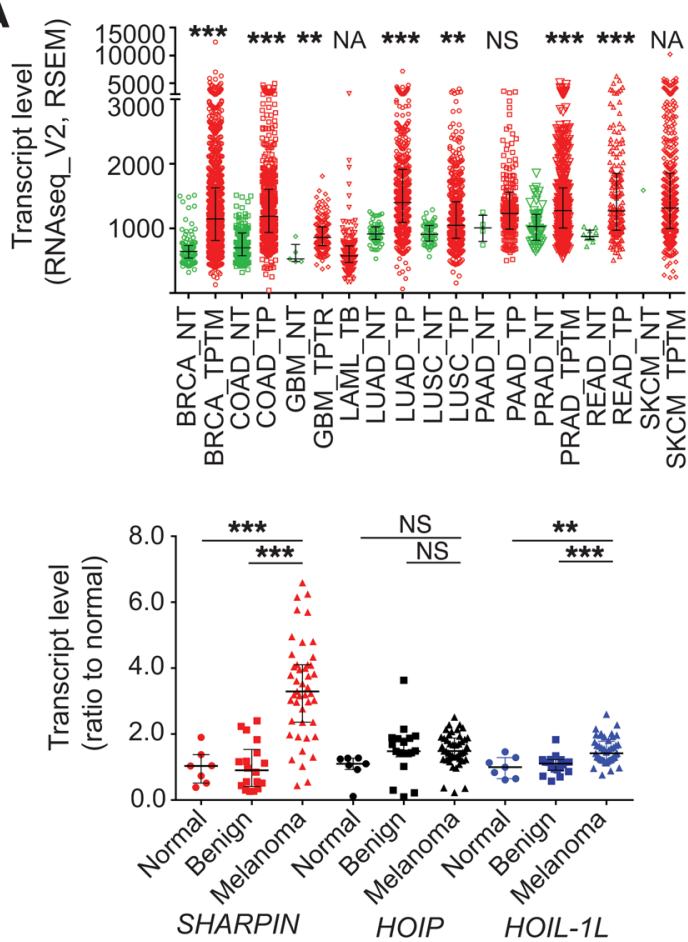

B
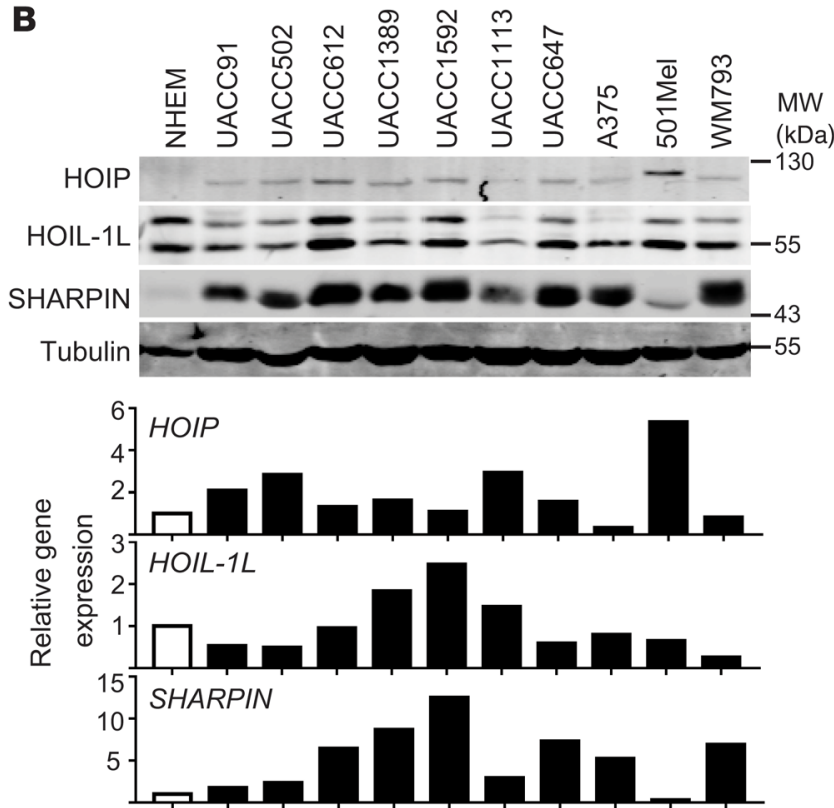

Figure 1. SHARPIN is upregulated in melanoma. (A) Upper panel: SHARPIN transcript levels in diverse cancer types (red dots, $n=$ approximately $169-$ 998, TCGA) and normal tissue (green dots, $n=$ approximately 0-155) (2-tailed Mann-Whitney U test). Lower panel: HOIP, HOIL-1L, and SHARPIN transcript levels in normal tissue $(n=7)$, benign nevi $(n=18)$, and melanoma $(n=45)$ (CDS1375 data set). One-way ANOVA with Dunnett's test. (B) Immunoblot (upper panel) and GPCR (lower panel) analysis of LUBAC components in melanocytes (NHEM) and the indicated melanoma cell lines. Tubulin was probed as a loading control. qPCR data are presented as relative mRNA levels compared with NHEM cells. Data are representative results from 2 experiments. ${ }^{* *} P<0.005$; ${ }^{* *} P<0.0005$. NA, not applicable.

including melanoma, compared with normal or benign tissues (Figure 1A and Supplemental Figure 1, A and B; supplemental material available online with this article; https://doi.org/10.1172/ JCI95410DS1). In addition, expression of SHARPIN, but not HOIL$1 L$ or HOIP, was elevated in most (13 of 16 ) human melanoma cell lines examined compared with normal human melanocytes (Figure $1 \mathrm{~B}$ and Supplemental Figure 1C), pointing to a possible role for SHARPIN in melanomagenesis. Indeed, shRNA-mediated knockdown (KD) of SHARPIN, and to a lesser extent HOIL and HOIP, attenuated the growth of many but not all melanoma cell lines (Figure 2, A and B, and Supplemental Figure 1D). The growth inhibition was rescued by SHARPIN reexpression (Figure 2D and Supplemental Figure 1G). Notably, KD of SHARPIN, but not of HOIP or HOIL-1, further enhanced the inhibition of melanoma survival and growth by etoposide treatment, even though NF- $\mathrm{BB}$ relocalization and phosphorylation were equally inhibited by KD of each protein (Supplemental Figure 1, E, F and H). These data support a potential LUBAC/NF-KB-independent contribution of SHARPIN to melanoma growth, which was further substantiated by the finding that expression of I $\mathrm{\kappa} \alpha \Delta \mathrm{N}$, a superrepressor of NF- $\mathrm{KB}$, had no effect on melanoma growth despite attenuating NF-кB activation (Supplemental Figure 1I). Importantly, melanoma growth in vivo was also markedly attenuated by SHARPIN KD (Figure 2C and Supplemental Figure 1J). Collectively, these observations suggest that a LUBAC/NF-kB-independent function of SHARPIN is important for the growth of a subset of melanomas.
SHARPIN affects key melanoma signaling pathways. Ectopic expression of SHARPIN led to upregulation of MITF, the master regulator of melanocyte biogenesis, as well as its upstream regulators SOX10 and PAX3 and its downstream pigmentation gene targets SILV, DCT, TYR, and MLANA (Figure 3A). Conversely, SHARPIN KD in both human and mouse melanoma cells attenuated the expression of these genes (Figure 3, B and C, and Supplemental Figure 2A), but had no effect on CREB or ATF2 transcription factors, which have also been implicated in the regulation of MITF (Supplemental Figure 2B) $(16,17)$. Reexpression of SOX10 (Figure 3D), but not of PAX3 (data not shown), in SHARPIN-depleted melanoma cells partially restored colony formation and attenuated the reduction of MITF expression (Supplemental Figure 2C), implicating SOX10 in SHARPIN-mediated control of melanoma growth. Our attempts to evaluate the effect of MITF reexpression in SHARPIN KD melanoma cell lines were inconclusive because MITF overexpression inhibited cell growth, as observed previously (18). The effects of SHARPIN are unlikely to be mediated via its reported regulation of PTEN (7), since SHARPIN expression levels do not correlate with AKT activation and SHARPIN KD does not affect AKT activity in melanoma cells regardless of their PTEN mutation status (Supplemental Figure 2, D and E). Thus, SOX10 regulation represents a previously unknown mechanism of SHARPIN-mediated control of melanoma cell survival and growth.

SHARPIN interacts with PRMT5. We performed liquid chromatography-tandem mass spectrometry (LC-MS/MS) to identify proteins that interact with SHARPIN in melanoma cells. These 
A
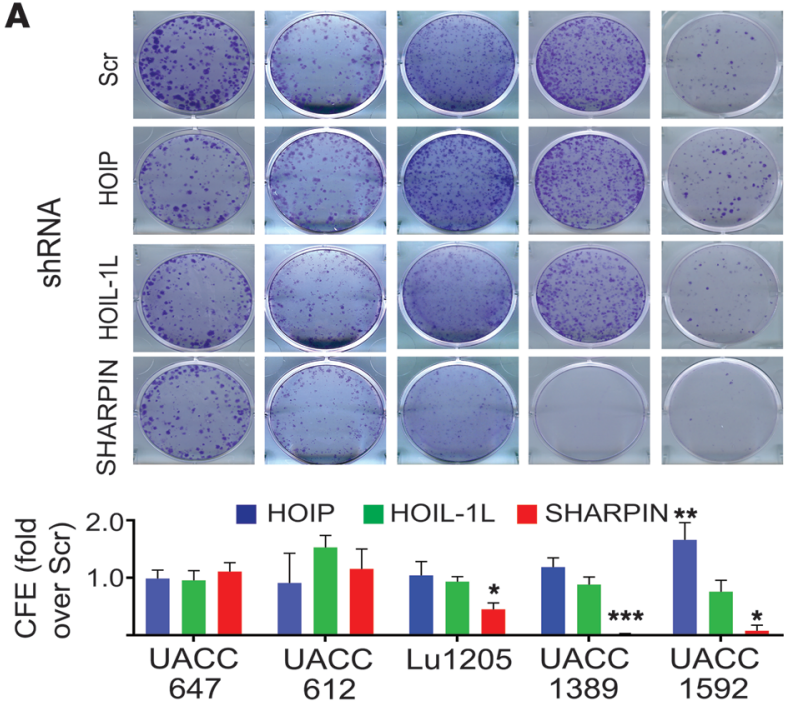

C

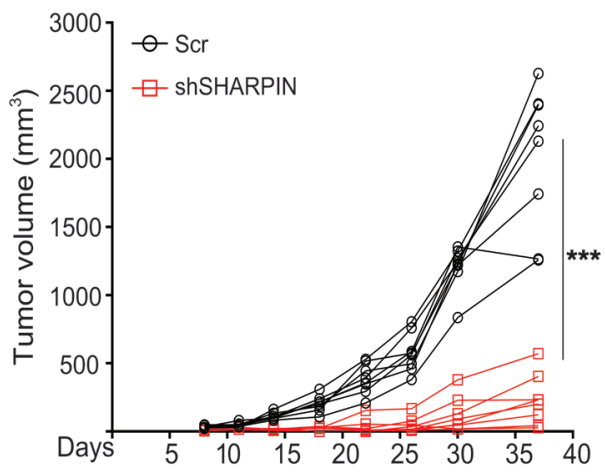

B
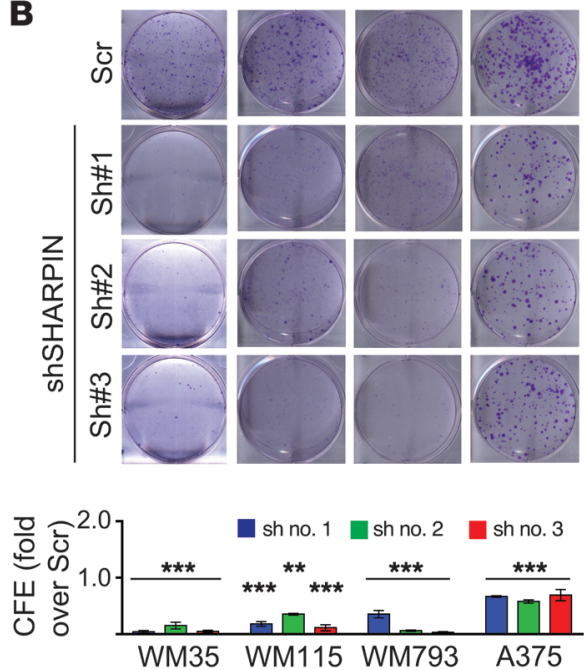

D
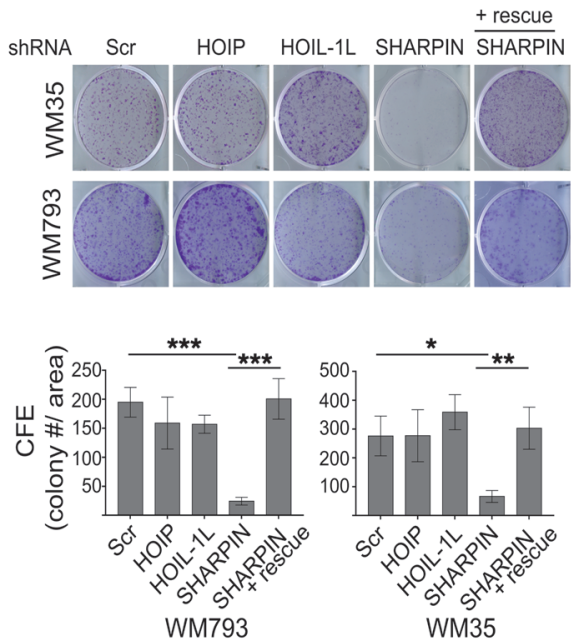

Figure 2. SHARPIN plays a role in melanoma growth. (A) Colony-forming efficiency (CFE) assay of melanoma cell lines expressing scrambled (Scr) or HOIP-, HOIL-1L-, or SHARPIN-specific shRNA. Cells were seeded at $10^{3} /$ well and incubated for 14 days. Upper panels show representative images on day 14 , and lower graph shows quantification of CFE (Image)). KD efficiency of each gene was confirmed with immunoblotting (see Supplemental Figure 1D). (B) CFE assay of melanoma cell lines expressing scrambled or SHARPIN-specific shRNAs (nos. 1, 2, and 3). Cells were seeded at $2.5 \times 10^{3} /$ well and analyzed as in D. (C) Growth of WM115 cells $\left(4 \times 10^{6}\right.$ cells) expressing scrambled or SHARPIN-specific shRNA after subcutaneous injection into female nude mice. Tumor volumes were measured at the indicated time points. Data are presented as mean \pm SD. $n=8$ mice/group. Statistical significance was calculated using 2 -way ANOVA. (D) CFE assay of WM793 and WM35 cells treated as indicated in C except that SHARPIN was reexpressed (+ rescue) as indicated. Upper panel shows representative images of colonies on day 14 after seeding at $10^{3} /$ well. Lower graph shows CFE quantification on day 14 . All quantitation data are presented as mean \pm SD $(n=3)$. (A, B, D) Statistical significance was calculated using 1-way ANOVA and Dunnett's test. ${ }^{*} P<0.05 ;{ }^{*} P<0.005 ;{ }^{* * *} P<0.0005(2$-tailed Student's $t$ test). (A and $\mathbf{B}$ ) Data are representative of 3 experiments.

experiments identified not only HOIP and HOIL-1, which was expected, but also a number of unreported proteins, including PRMT5/MEP50 (Supplemental Table 1). SHARPIN binding to the PRMT5/MEP50 complex was confirmed through a series of coimmunoprecipitations of endogenous proteins in melanoma cells (Figure 4A and Supplemental Figure 3A). In addition, SHARPIN coeluted with the PRMT5/MEP50 complex and with HOIP in gel filtration fractions of melanoma cell lysates (Figure 4B and Supplemental Figure 3B). Two-dimensional PAGE analysis also confirmed that SHARPIN was present in PRMT5 complexes (Figure 4C). To map the interacting domains in SHARPIN and PRMT5, we coexpressed V5-tagged PRMT5 with Flag-tagged SHARPIN
WT protein or mutants lacking 1 of the 3 major SHARPIN domains (ubiquitin-binding domain [UBL], coiled-coil domain [CC], or Npl4 zinc finger domain [NZF]). Coimmunoprecipitation experiments showed that the SHARPIN UBL domain, but not the NZF or CC domains, was required for interaction with PRMT5 (Figure 4D and Supplemental Figure 3C). Full-length HOIL-1L did not bind to PRMT5, despite the sequence similarity of the SHARPIN and HOIL-1L UBL domains (Supplemental Figure 3D). These observations confirm that the SHARPIN UBL-PRMT5 interaction is specific and suggest that it may depend on a conformation dictated by proximal domains or by specific posttranslational modifications (19) (Supplemental Figure 3D). HOIP, which is known to interact 
A

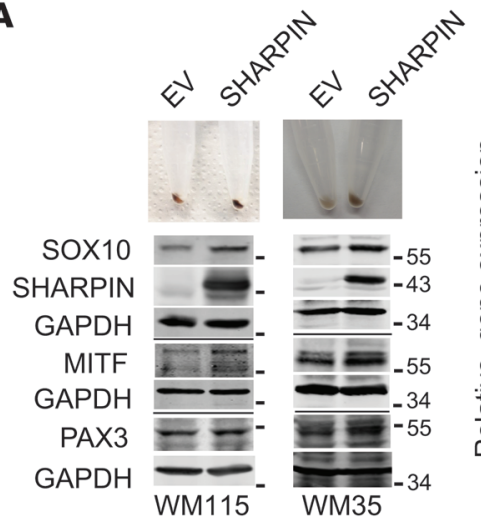

C

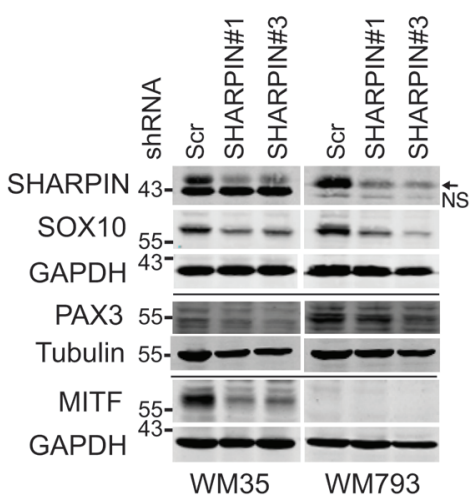

B

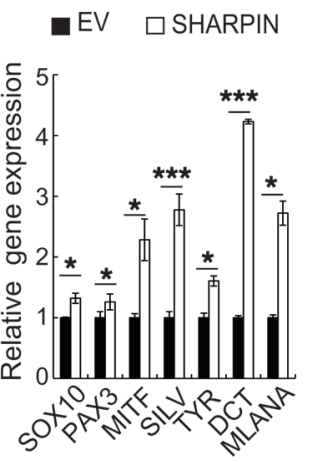

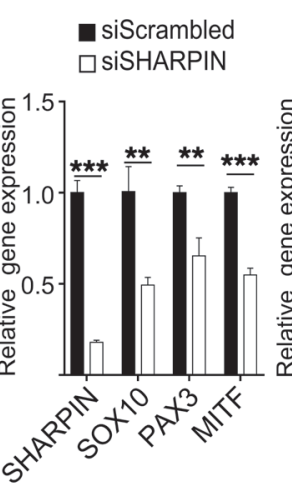

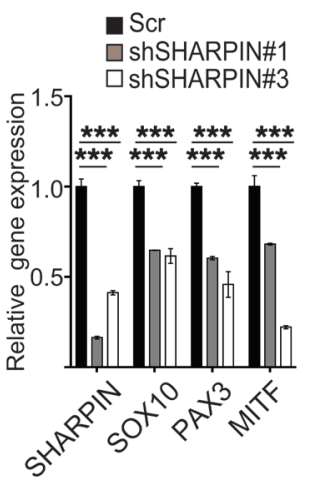

$\mathbf{E}$
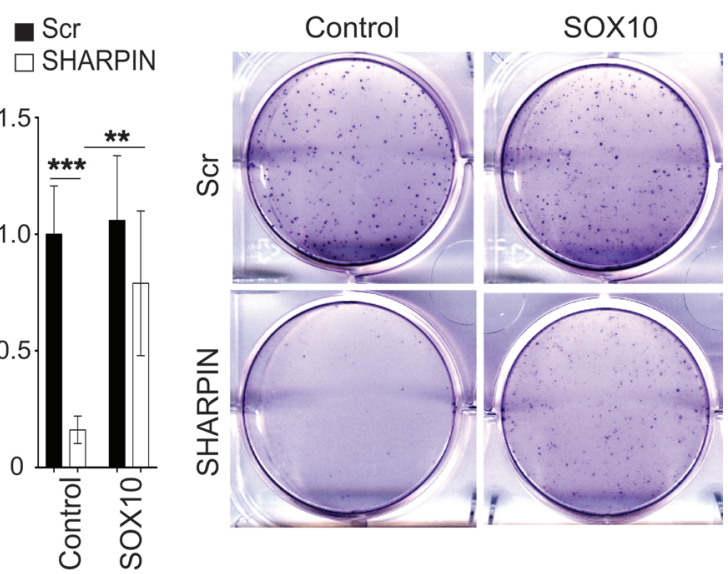

Figure 3. SHARPIN affects melanoma growth and survival through regulation of SOX10, PAX3, and MITF expression. (A) Pigmentation (upper panels), immunoblot (lower panels), and qPCR (right panel) analysis of MITF and upstream/downstream regulatory proteins in WM115 or WM35 melanoma cells overexpressing SHARPIN or a control empty vector (EV). qPCR data are presented as relative mRNA levels compared with control cells. (B) Immunoblot (left panel) and qPCR (right panels) analysis of WM115 cells expressing scrambled or SHARPIN-specific siRNAs or shRNAs. Statistical significance was calculated using Student's $t$ test (unpaired, 2 tailed, siRNA) or 1-way ANOVA and Dunnett's test (shRNA). (C) Immunoblot analysis of WM35, WM793 and WM115 cells expressing scrambled or SHARPIN-specific shRNAs. Arrow and NS indicate SHARPIN and nonspecific bands, respectively. (D) Immunoblot analysis and CFE assay of WM115 melanoma cells expressing scrambled or SHARPIN-specific shRNA and ectopically expressing control or SOX10 (rescue). Middle and right panels show representative images of colonies and quantification of CFE 14 days after cells were seeded at $10^{3} /$ well. CFE (ratio to scrambled/control) was presented as mean \pm SD, and statistical significance was calculated using 2-way ANOVA and Tukey's test ( $n=6$ from 2 independent experiments). All qPCR data are presented as mean $\pm \mathrm{SD}(n=3){ }^{*} P<0.05 ;{ }^{* *} P<0.005 ;{ }^{* *} P<0.0005$ (2-tailed, Student's $t$ test). (A-D) Data are representative of 2 experiments.

with the SHARPIN UBL domain, did not compete with PRMT5 for binding to SHARPIN (Supplemental Figure 3E). Experiments with cells expressing differentially tagged $\mathrm{N}$ and $\mathrm{C}$ termini of PRMT5 demonstrated that both portions interacted with the UBL domain of SHARPIN (Figure 4E and Supplemental Figure 3F). Notably, these experiments also revealed that SHARPIN increases the interaction between the $\mathrm{N}$ and $\mathrm{C}$ termini of PRMT5, pointing to a possible effect on intramolecular interactions in the PRMT5 complex $(20,21)$ (Supplemental Figure 3G).

SHARPIN regulation of PRMT5 activity affects SOX1O and MITF. Protein arginine methylation by PRMT5/MEP50 plays an important role in epigenetic regulation of histones and transcription factors (10). Although PRMT5 has previously been shown to affect MITF expression and melanoma growth (22), the underlying mechanisms were unknown. Therefore, we investigated whether PRMT5 plays a role in SHARPIN-mediated control of SOX1O and PAX3 transcription and melanoma growth. We first determined how binding to SHARPIN affects the methyltransferase activity of the PRMT5/MEP50 complex. KD of SHARPIN attenuated the enzymatic activity of PRMT5, as measured by symmetric dimethylation of arginine (SDME-RG) on a histone 4 peptide substrate (Supplemental Figure 3H). Consistent with this, expression of the PRMT5-regulated genes $S T 7$ and RBL2, but not NM23, was elevated upon SHARPIN KD. The increase in ST7 expression was accompanied by reduced SDME of histone 4 arginine 3 (H4R3me2s) at the enhancer/promoter region $(23,24)$ (Figure 4F). Of note, we did not observe global changes in SDMERG in histone H4 upon SHARPIN KD (Supplemental Figure 3I), suggesting that SHARPIN regulation of PRMT5 affects a selected subset of target genes (e.g., ST7 but not NM23). Consistent with this, SHARPIN KD and overexpression significantly decreased and increased, respectively, SDME-RG of a subset of nonhistone proteins (Figure 4G and Supplemental Figure 3J). In vitro assays confirmed that methylation of $\mathrm{H} 4$ protein and $\mathrm{H} 4$ peptide by 
A

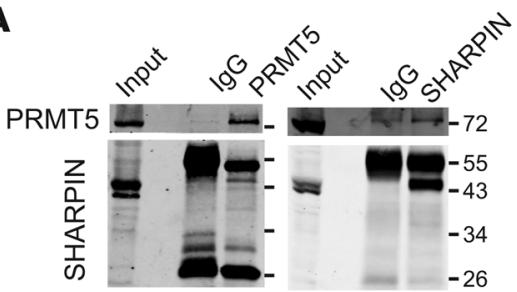

B

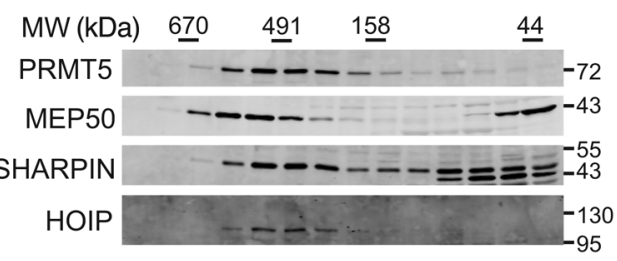

C

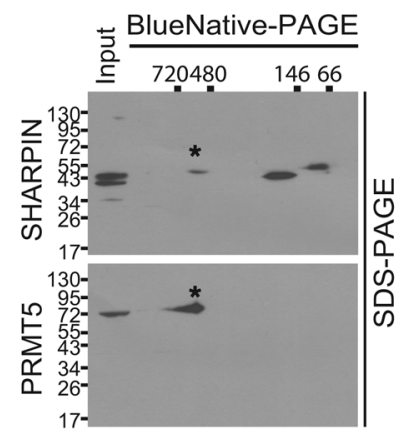

D

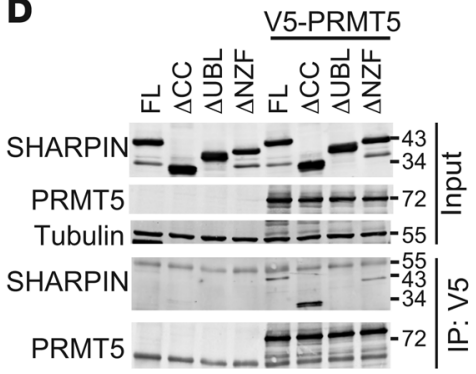

E

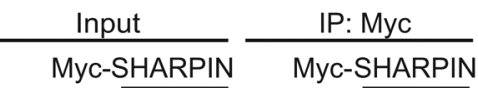

E๗

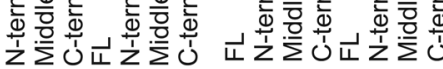

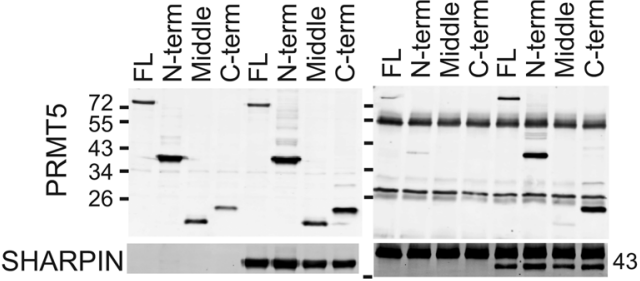

$\mathbf{F}$

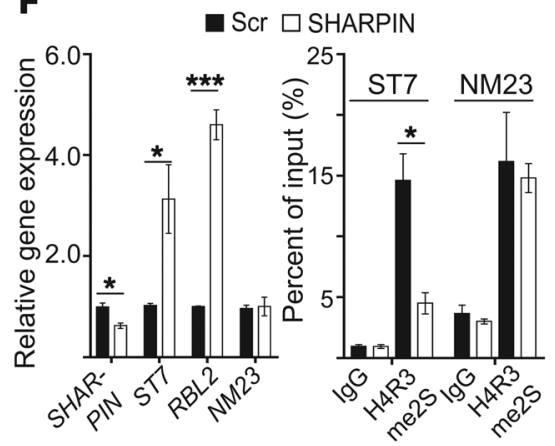

G

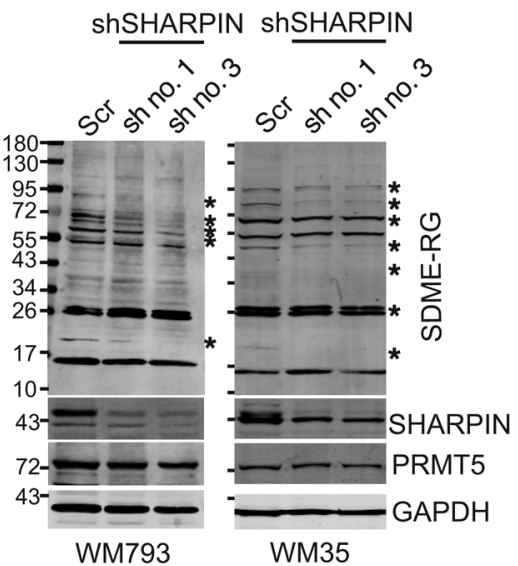

H

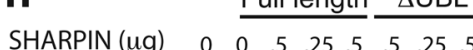
PRMT5/MEP50 $-+{ }_{-}+t_{-}+$ Histone 4

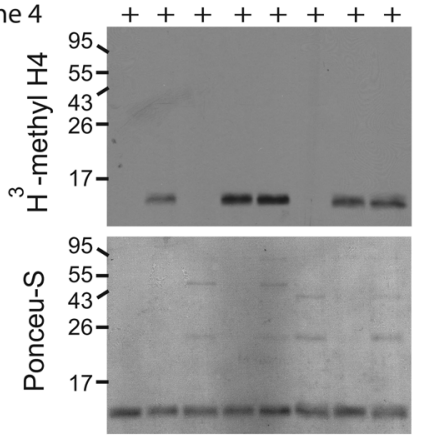

I

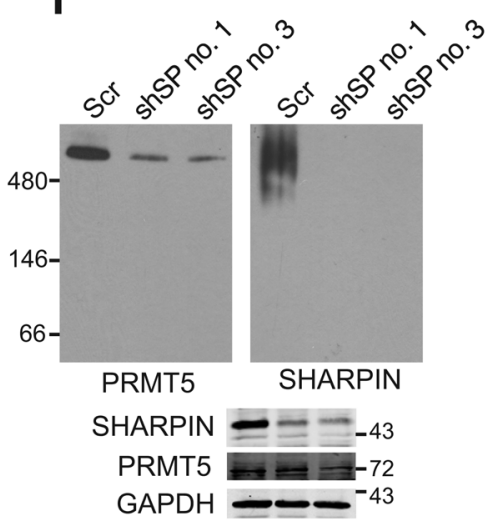

Figure 4. SHARPIN facilitates PRMT5 complex formation and enhances PRMT5 methyltransferase activity. (A) Immunoblot analysis of WM115 cell lysates immunoprecipitated with normal rabbit IgG, anti-PRMT5, or anti-SHARPIN antibodies and blotted for PRMT5 or SHARPIN. (B) Immunoblot analysis of gel filtration fractions from WM793 lysates (3 mg protein). (C) 2D Blue Native-PAGE/SDS-PAGE analysis of WM793 lysates (40 $\mu \mathrm{g} / \mathrm{lane}$ ). Asterisks indicate SHARPIN and PRMT5 in the complex. (D) Immunoblot analysis of V5-immunoprecipitates from HEK293T cells expressing empty vector or V5-tagged PRMT5 and coexpressing Myc-tagged SHARPIN full-length (FL), NZF-deleted ( $\triangle N Z F$ ), UBL deleted ( $\triangle U B L$ ), or Flag-tagged CC-deleted ( $\triangle C C$ ) proteins. (E) Immunoblot analysis of Myc immunoprecipitates from HEK293T cells expressing empty vector or Myc-tagged full-length SHARPIN and coexpressing Flag-tagged full-length PRMT5, N terminus (N-term), middle region (middle), and C terminus (C-term). (F) qPCR analysis (left) of SHARPIN and the PRMT5 target genes ST7, RBL2, or NM23 in WM35 cells expressing scrambled or SHARPIN-specific shRNA. ChIP analysis (right) of arginine-methylated histone $\mathrm{H} 4$ at the enhancer/promoter of ST7 or NM23 genes. WM35 cells expressing scrambled or SHARPIN-specific shRNA were immunoprecipitated with an anti-H4R3me2s antibody, and coimmunoprecipitated ST7 or NM23 promoter sequences were quantified by qPCR. (G) Immunoblot analysis of WM793 or WM35 cells expressing scrambled or SHARPIN-specific shRNAs (nos. 1 and 3). (H) In vitro methylation of histone 4 by PRMT5/MEP50 after preincubation with buffer or purified full-length or $\triangle$ UBL SHARPIN proteins. (I) Blue Native-PAGE gel of lysates from WM793 cells expressing scrambled or SHARPIN-specific shRNAs (nos. 1 and 3). For immunoblot/immunoprecipitation analyses, input indicates $5 \%$ of lysate. qPCR data are presented as mean $\pm \mathrm{SD}(n=3) .{ }^{*} P<0.05 ;{ }^{* *} P<0.005 ;{ }^{* *} P<0.0005$, 2-tailed Student's $t$ test. (A-I) Data shown represents results of at least 2 independent experiments.

recombinant PRMT5/MEP50 was induced by full-length SHARPIN, but not by the $\triangle$ UBL mutant protein (Figure $4 \mathrm{H}$ and Supplemental Figure 3, $\mathrm{K}$ and $\mathrm{L}$ ). SOX10, PAX3, and MITF expression were elevated upon ectopic expression of WT SHARPIN, but not by expression of $\triangle$ UBL SHARPIN (Supplemental Figure 3M).
PRMT5 activity is regulated through various mechanisms, including multimerization, subcellular localization, association with MEP50 and other cofactors, and posttranslational modification (20, 21). Since SHARPIN interacts with MEP50, but does not cooperate or compete with it for binding to PRMT5 (Supplemental Figure 3, 
A

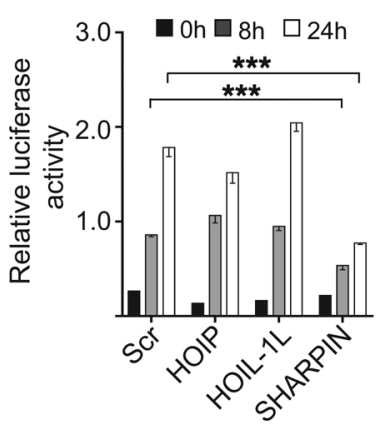

D

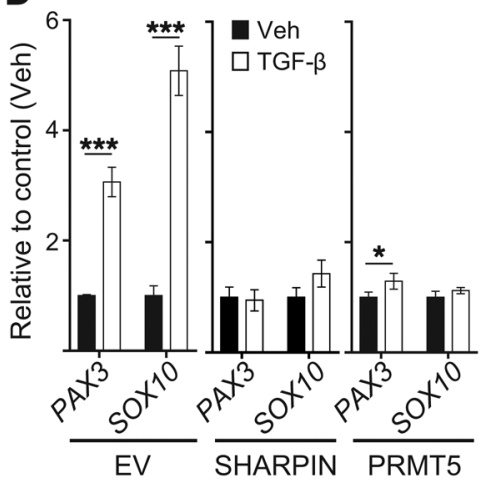

B

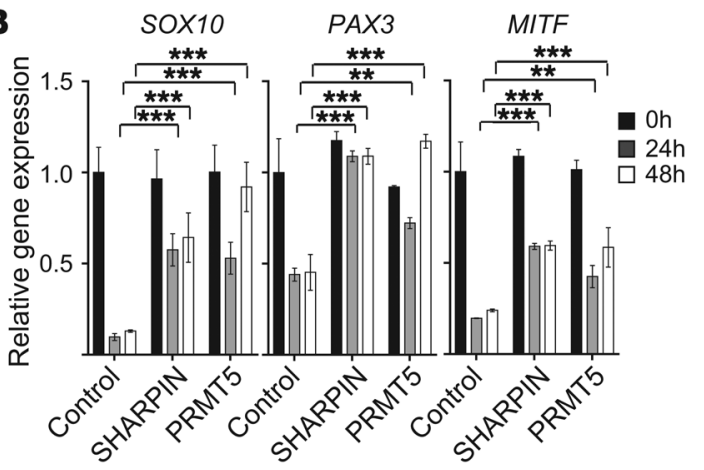

E
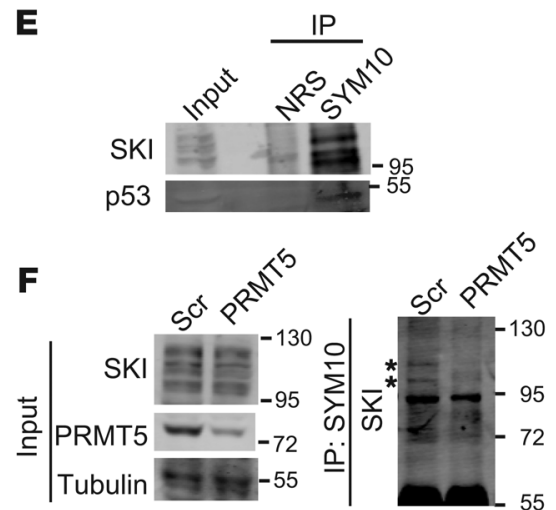

G
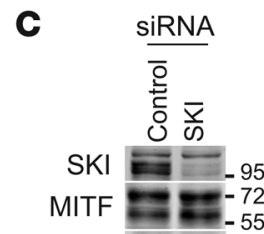

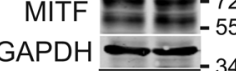

SOX10

PAX3

GAPDH
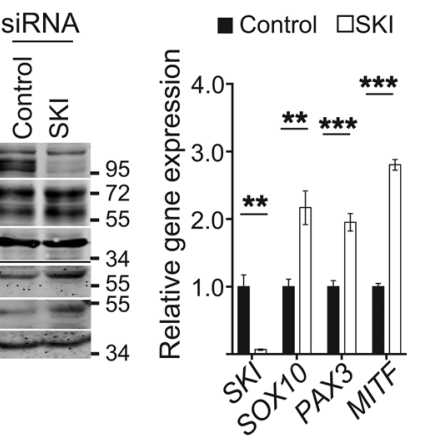

H

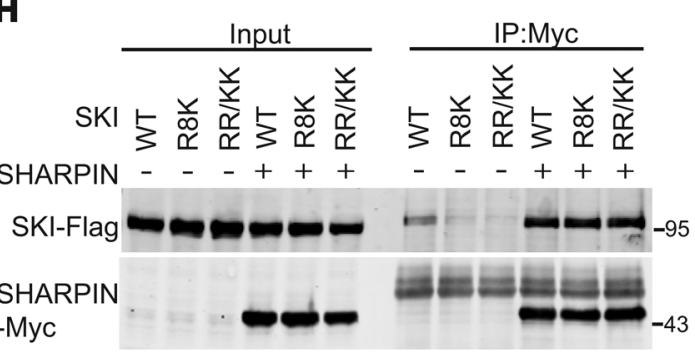

I

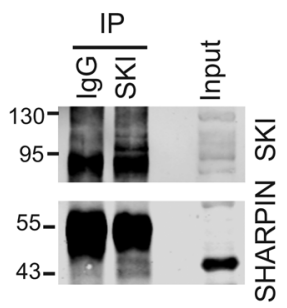

J

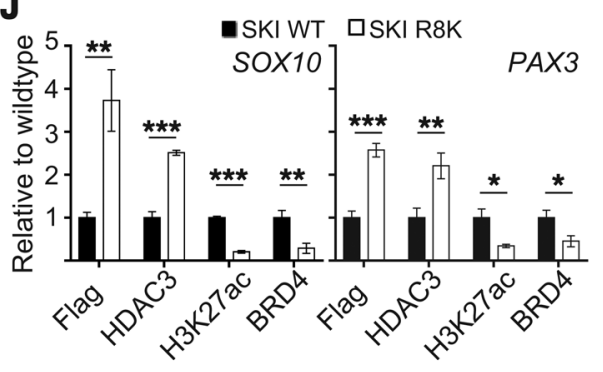

Figure 5. PRMT5 increases SOX10 and PAX3 expression by arginine methylation-dependent inhibition of SKI. (A) Luciferase reporter assay of TCF- $\beta$ signaling in WM793 cells expressing scrambled shRNA or HOIP-, HOIL-1L-, or SHARPIN-specific shRNAs. Cells transfected with TCF- $\beta$ reporter (CACAx9luc) and pCMV-Cypridina-luc (internal control) reporter plasmids were treated with TGF- $\beta$ ( $20 \mathrm{ng} / \mathrm{ml})$ for 8 or 24 hours. (B) qPCR analysis of SOX10, PAX3, and MITF expression in WM115 cells expressing empty vector (control), SHARPIN, or PRMT5 expression plasmids and treated with TCF- $\beta$ (20 ng/ml) for 0,24 , or 48 hours. (C) Immunoblot and qPCR analysis of WM115 cells transfected with scrambled (control) siRNA or SKI-specific SMARTpool siRNA. (D) ChIP analysis of WM115 cells expressing empty vector, SHARPIN, or PRMT5 and treated with vehicle (Veh) or TGF- $\beta$ (20 ng/ml) for 18 hours. ChIP analysis was performed with anti-SKI antibody, and coimmunoprecipitated SOX10 or PAX3 promoter sequences were quantified by qPCR. (E) Immunoblot analysis of SKI and p53 in WM115 cell lysates immunoprecipitated with normal rabbit serum (NRS) or SYM10 antibody. (F) Analysis as in E except WM115 cells expressed scrambled or PRMT5-specific shRNA. (C) Immunoblot analysis of anti-Flag immunoprecipitates of A375 cells coexpressing WT, Arg8 mutated (R8K), or Arg658/660 mutated (RR/KK) SKI with either PRMT5 or SHARPIN. Asterisks and arrowhead indicate SHARPIN and tubulin, respectively. (H) Immunoblot analysis of anti-Myc immunoprecipitates of HEK293T cells expressing Myc-tagged SHARPIN and Flag-tagged WT, R8K mutant, or RR/KK mutant SKI proteins. (I) Immunoblot analysis of IgC or anti-SKI immunoprecipitates of SK-Mel-28 cells. (J) ChIP analysis as in D of WM115 cells expressing WT or R8K mutant SKI proteins. Lysates were immunoprecipitated with indicated antibodies. For immunoprecipitation analyses, input indicates $5 \%$ of lysates. Statistical significance was calculated using 2-way ANOVA (Dunnett's test, $\mathbf{A}$ and $\mathbf{B}$ ) or 2-tailed Student's $t$ test (C, D, J). Data are presented as mean \pm SD $(n=3) .{ }^{*} P<0.05 ;{ }^{* *} P<0.005 ;{ }^{* *} P<0.0005$.

$\mathrm{N}-\mathrm{P}$ ) or affect PRMT5 expression levels or cellular localization (Supplemental Figure 3, Q and R), we asked whether SHARPIN influences the formation of PRMT5/MEP50 multiprotein complexes. In support of this possibility, there is biochemical and structural evidence that PRMT5 activity can be elevated by multiprotein complex formation $(20,21)$. This is consistent with our observation that SHARPIN enhanced intramolecular interactions between the PRMT5 N and C termini (Supplemental Figure 3G) and that endogenous SHARPIN coeluted with the endogenous PRMT5 complex $(-500 \mathrm{kDa}$; Figure 4, B and C, and Supplemental Figure 3B). Ectopic expression of full-length SHARPIN, but not the $\triangle$ UBL mutant, in HEK293T cells increased the levels of an approximately 500-kDa PRMT5-SHARPIN complex (Supplemental Figure 3S). Moreover, SHARPIN KD and overexpression decreased and increased, respectively, levels of the 

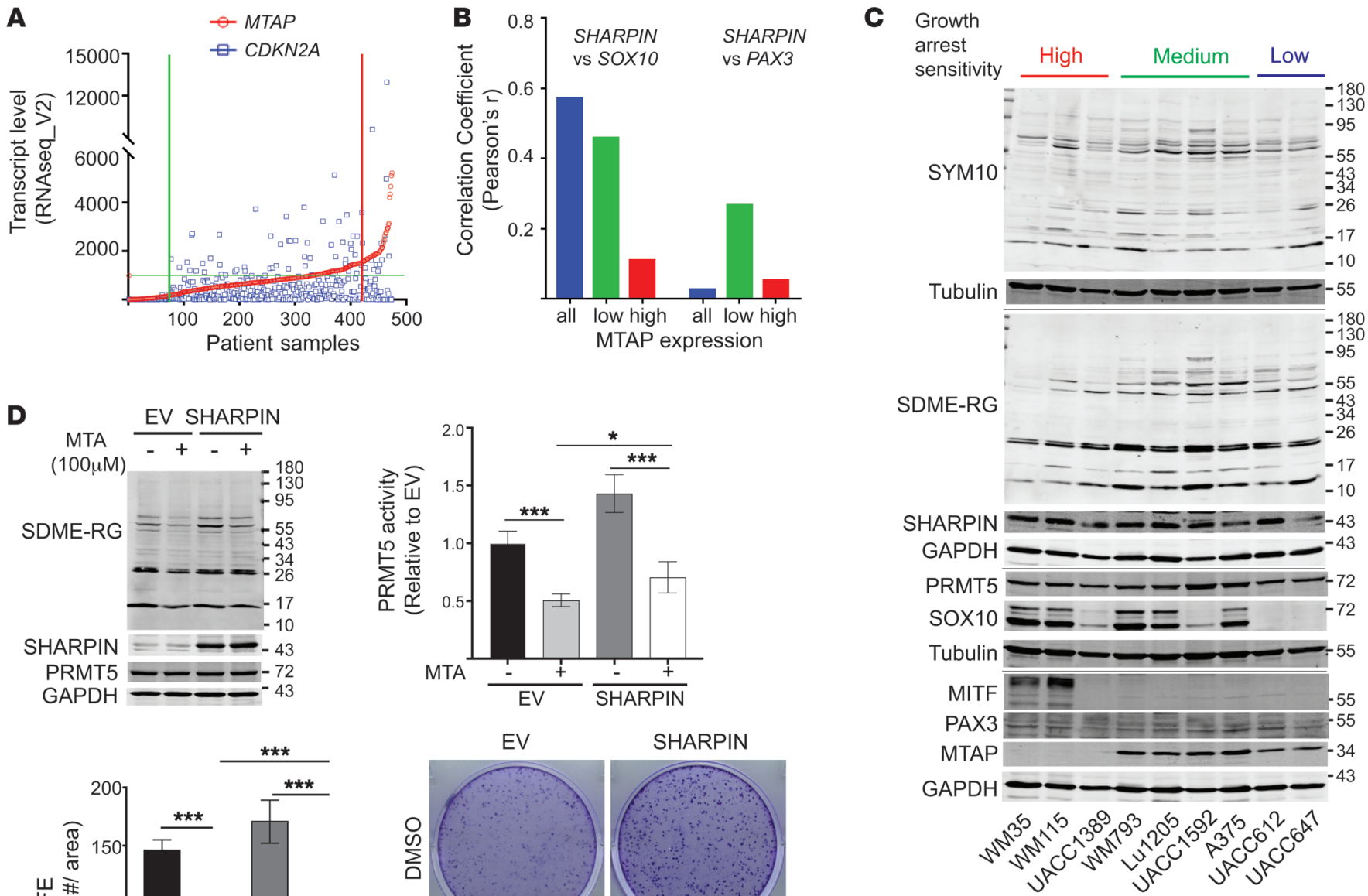
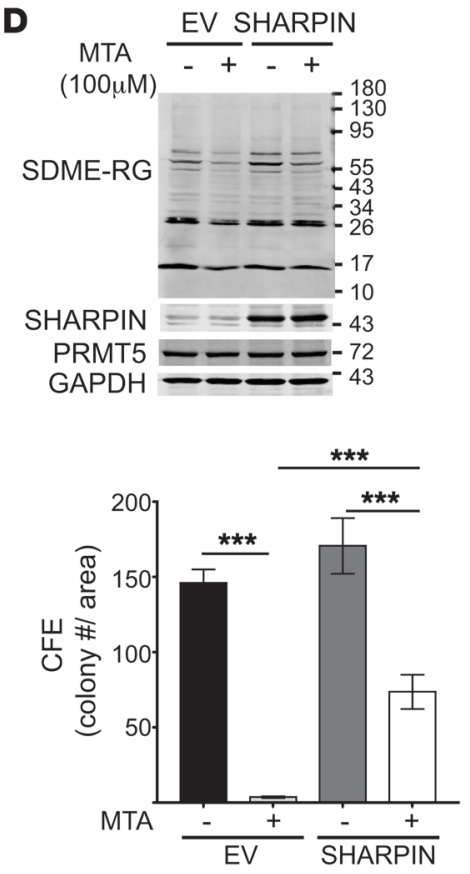
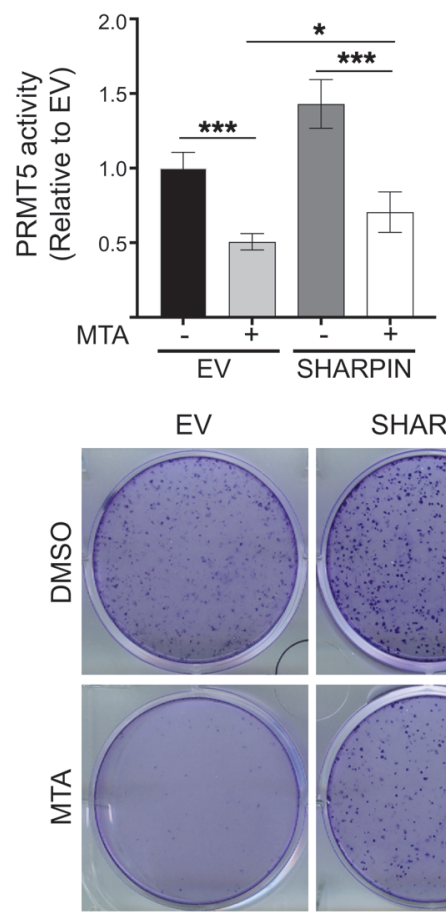

SHARPIN

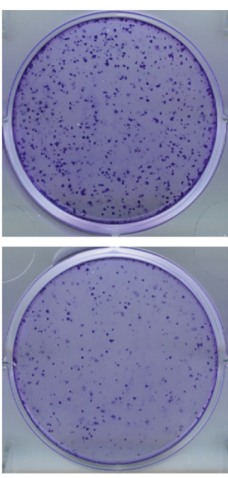

Figure 6. SHARPIN regulation of PRMT5 activity controls SOX10 expression and growth in MTAP-deleted human melanomas. (A) Expression of MTAP and CDKN2A genes in melanoma patients (TCGA, $n=472$ ). Green and red vertical lines indicate the cutoffs for classification as low-MTAP ( $n=70)$ and high-MTAP $(n=50)$ melanomas, respectively. The green horizontal line indicates the expression level in normal tissue (TCGA, $n=1)$. (B) Pearson's correlation coefficients for SHARPIN and SOX10 or SHARPIN and PAX3 expression in all ( $n=472$, blue), MTAP-low ( $n=70$, green), and MTAP-high ( $n=50$, red) melanoma samples. (C) Immunoblot analysis of the indicated proteins in melanoma cells with high, medium, or low sensitivity to growth inhibition by SHARPIN KD. (D) Immunoblot analysis (left upper) and colony-forming efficiency (lower) assay of WM35 cells expressing empty vector (control) or overexpressing SHARPIN. Cells were treated with vehicle (DMSO) or MTA $(100 \mu \mathrm{M})$ for 72 hours. For CFE, cells were seeded at $10^{3} /$ well and colonies were visualized and quantified (lower right) after 14 days in culture. PRMT5 activity (upper right) was assessed in anti-PRMT5-immunoprecipitated cell lysates. The quantitation data are presented as mean \pm SD. $n=3$ (lower right). $n=6$ (upper right). Statistical significance was calculated using 2-way ANOVA (Tukey's test). ${ }^{*} P<0.05 ;{ }^{* *} P<0.0005$. (C and $\left.\mathbf{D}\right)$ Data shown represent at least 2 independent experiments.

PRMT5 complex (Figure 4I and Supplemental Figure 3, T and U). Thus, binding of SHARPIN to PRMT5 through the SHARPIN UBL domain appears to enhance the formation or stabilization of PRMT5 multiprotein complexes, thereby increasing PRMT5 activity and/or substrate-specific methylation in melanoma cells.

We next determined whether SHARPIN regulation of PRMT5 activity affects the expression of SOX1O and PAX3 genes. SOX10, PAX3, and MITF expression were significantly reduced by PRMT5 KD (Supplemental Figure 3V) and upregulated upon ectopic expression of WT PRMT5, but not a catalytic mutant (E444Q) (Supplemental Figure 3X). Furthermore, PRMT5 depletion inhibited the growth of SHARPIN-sensitive WM35 cells (Supplemental Figure 3W), indicating that PRMT5 KD phenocopies SHARPIN KD (Figure 2, A and B). These observations suggest that the interaction between SHARPIN and PRMT5/MEP50 facilitates formation of multimeric complexes. In turn, the increase in PRMT5 activity upregulates SOX10 and PAX3 expression and promotes melanoma growth.

SHARPIN-PRMT5 effect on SOX1O signaling is mediated by SKI. To identify the protein or proteins that may mediate the PRMT5 effect on PAX3 and SOX10 expression, we examined the possible involvement of the TGF- $\beta$ signaling pathway, which has been implicated in the negative regulation of PAX3 and $\operatorname{SOX} 10(25,26)$. Con- 
A

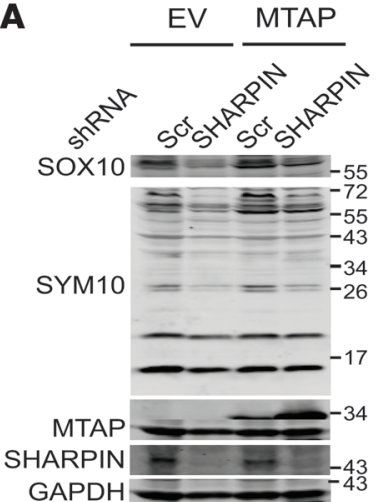

B
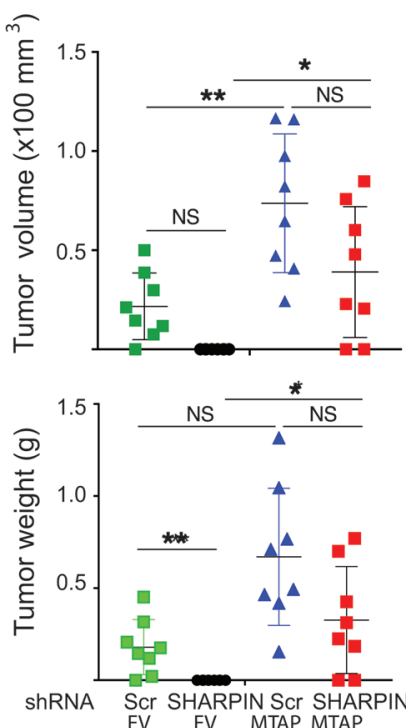

Scr SHARPIN Scr SHARPI
EV EV MTAP MTAP

E

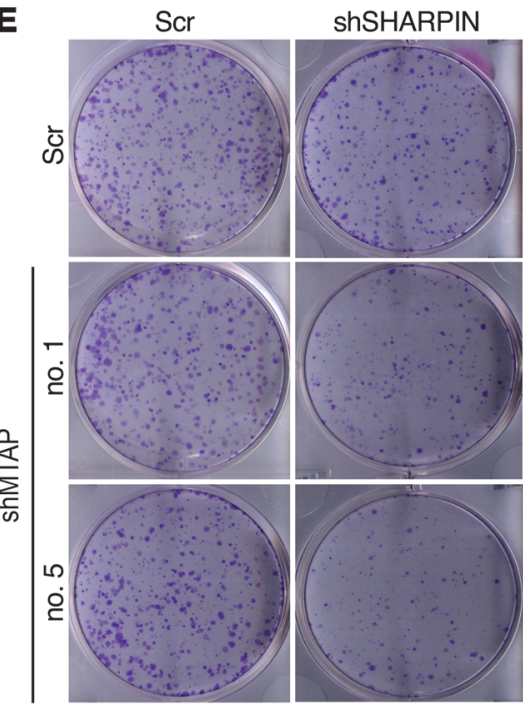

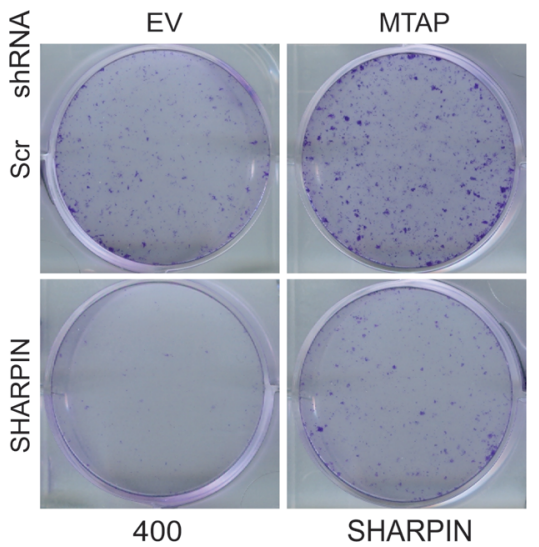

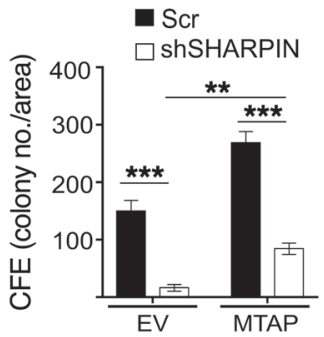

C
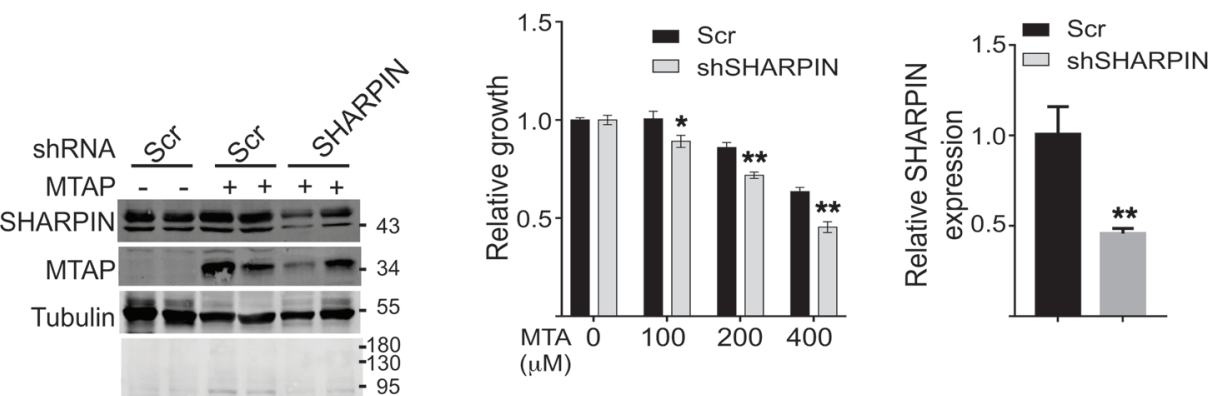

D
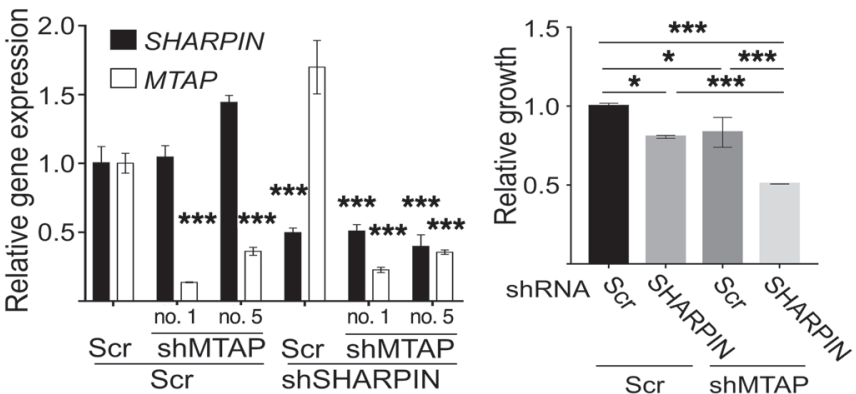

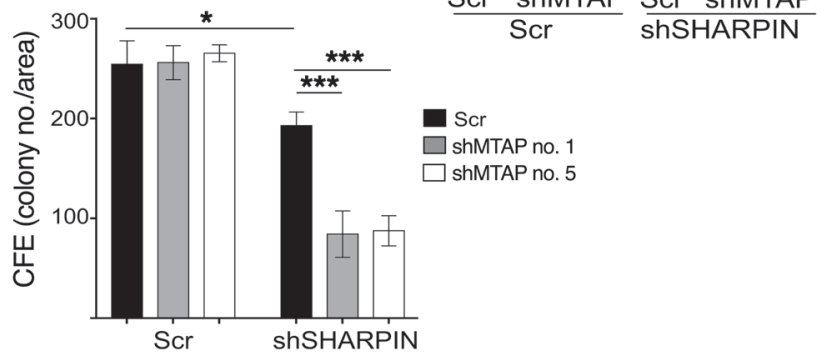

Figure 7. MTAP expression determines sensitivity to SHAPIN-mediated growth inhibition of melanoma. (A) Immunoblot analysis and CFE assay of WM35 cells stably expressing empty vector or MTAP expression plasmids plus scrambled or SHARPIN-specific shRNA. Cells were seeded at $10^{3} /$ well and colonies were visualized and quantified after 14 days in culture. (B) Growth of WM35 cells $\left(2.0 \times 10^{6}\right.$ cells) injected subcutaneously into female nude mice. Stable WM35 transfectants expressing empty vector or MTAP were subjected to KD with scrambled or SHARPIN-specific shRNA. Tumor volume (upper panel) and weight (lower panel) were measured at the indicated time. Immunoblot analysis (right panel) was performed using representative tumor lysates from each group. Data are presented as mean \pm SD (at the end point of experiment). $n=8$ mice/group. Two-way ANOVA test (tumor volume) or Welch's test (tumor weight). (C) Cell growth/ viability (ATPlite, left panel) of A375 cells expressing scrambled or SHARPIN shRNA, and then treated with indicated amount of MTA for 5 days. KD efficiency of SHARPIN was analyzed with qPCR (right panels). (D) As in C, qPCR analysis (left) and growth/viability assay (ATPlite, right) of A375 cells expressing shRNAs, scrambled, shSHARPIN, and/or shMTAP. (E) CFE assay of A375 cells expressing scrambled, SHARPIN, or MTAP (nos. 1 and 5) shRNAs. Cells ( $2.5 \times 10^{3} /$ well) were seeded and cultured for 14 days. Colonies were visualized and quantified (Image)). All quantitation data and qPCR data are presented as mean \pm SD $(n=$ 3). Statistical significance was calculated using 1-way (D, left) or 2-way (A and B, D, right, E) Tukey's test. ${ }^{*} P$ $<0.05$; ${ }^{* *} P<0.05$; ${ }^{* *} P<0.0005$. (A, C-E) Data represent results from 2 to 3 independent experiments. 

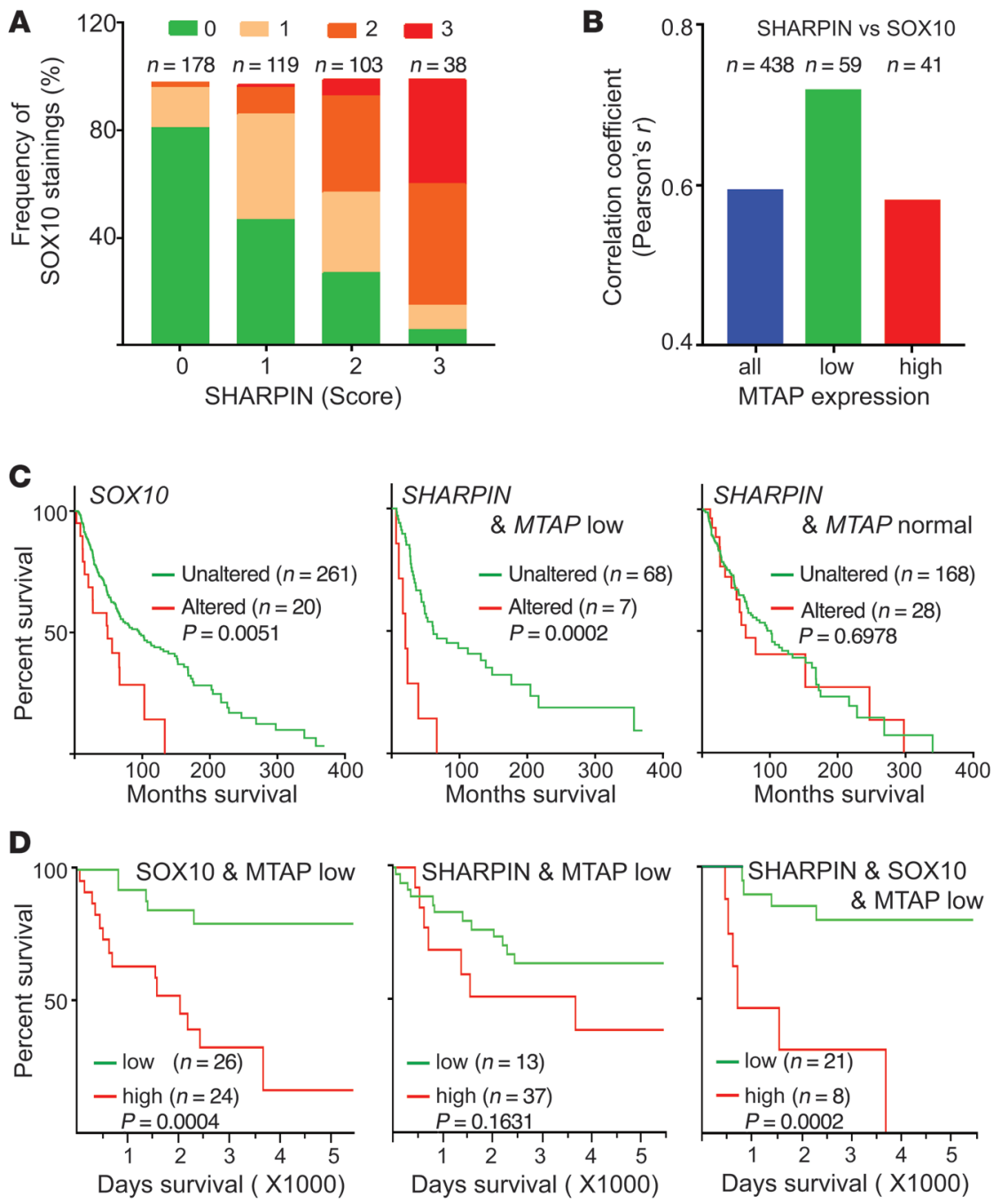

Figure 8. SHARPIN expression in MTAP-deleted tumors correlates strongly with SOX10 expression and patient survival. (A) Quantification of immunohistochemical staining of SHARPIN and SOX10 in a human melanoma tissue microarray ( $n$ $=438$ ). Staining intensity was scored as $0,1,2$, or 3 (see Supplemental Figure 6), and the data are expressed as the percentage of specimens with the indicated combinations of staining scores. (B) Pearson's correlation coefficients for SHARPIN and SOX10 expression in all, low-MTAP, and high-MTAP melanomas. (C) Survival of patients from the TCGA data set ( $n=281$ completely sequenced tumors). Left: patients with tumors expressing normal (unaltered) or amplified/upregulated (altered) SOX10 levels $(n=281)$. Middle: patients with tumors expressing unaltered or altered SHARPIN levels and MTAP deletion $(n=75)$. Right: patients with tumors expressing unaltered or altered SHARPIN levels and normal MTAP levels $(n=196)$. (D) Survival of patients from the TMA data set ( $n$ = 50). Patients with tumors expressing low MTAP (IHC score $=0$ or 1 ) and low (IHC score $=0$ or 1$)$ or high (IHC score $=2$ or 3 ) levels of SOX10 (left, $n=$ 50), SHARPIN (middle, $n=50$ ), or both SHARPIN and SOX10 (right, $n=29$ ). Statistical significance was calculated using Prism7.0 (log-rank test). sistent with earlier reports, treatment of melanoma cells with TGF- $\beta$ reduced SOX10 and PAX3 expression in a dose-dependent manner (Supplemental Figure 4A). KD of SHARPIN, but not of HOIP or HOIL-1L, reduced the activity of a TGF- $\beta$-driven (CAGA)9-luciferase reporter, suggesting that SHARPIN may negatively regulate TGF- $\beta$-dependent signaling (Figure $5 \mathrm{~A}$ ). Indeed, ectopic expression of either SHARPIN or PRMT5 overcame the TGF- $\beta$-mediated repression of SOX10, PAX3, and MITF (Figure 5B). Because the TGF- $\beta$ pathway components SKI and SNO are thought to regulate SOX10 and PAX3 expression and have been shown to interact with PRMT5 $(25,27)$, we examined SKI/SNO involvement in SHARPIN-PRMT5 regulation of gene expression. SKI KD increased the basal expression levels of SOX10, PAX3, and MITF (Figure 5C), and conversely, ectopic expression of WT SKI, but not SKI carrying a defective nuclear localization signal (28), decreased SOX10, PAX3, and MITF mRNA and protein levels (Supplemental Figure 4B). Consistent with these findings, ectopic expression of SHARPIN and PRMT5 inhibited SKI recruitment to SOX1O and PAX3 enhancers/ promoters following TGF- $\beta$ stimulation (Figure 5D).

To determine whether SKI mediates the PRMT5-dependent effects on SOX10 and PAX3, we first examined their interactions. SKI was present in PRMT5 immunoprecipitates from HEK293T cells (Supplemental Figure 4C) and coeluted with the PRMT5 com- plex and SHARPIN upon gel filtration of cell lysates (Supplemental Figure 4D). Immunoprecipitation of melanoma cells with antibodies specific for SDME-RG-containing proteins detected SKI (Figure 5E) and p53, consistent with previous reports (11). Moreover, arginine methylation of SKI was decreased following PRMT5 KD (Figure 5F). Although ectopic expression of PRMT5 in HEK293T cells slightly increased SKI methylation, we could not detect SKI methylation by PRMT5 in vitro (Supplemental Figure 4E). However, methylated SKI was detected in immunoprecipitates from cells expressing both SKI and SHARPIN, supporting a role for SHARPIN in SKI methylation (Supplemental Figure 4F). PRMT5 methylates arginine residues located in GRG motifs, of which 2 are present in SKI (R8 and R658/660, Supplemental Figure 4G) (29). Mutation of $\mathrm{R} 8$, but not of R658/660, attenuated SKI methylation in melanoma cells expressing SKI and SHARPIN, but not SKI and PRMT5 (Figure $5 G)$. Although SHARPIN can bind to SKI regardless of its arginine mutation status (Figure 5, H and I), we observed that coexpression of SHARPIN with the R8 SKI mutant, but not with WT or R658/660 mutant SKI, decreased SOX10 and, to a lesser degree, PAX3 expression (Supplemental Figure 4, H and I).

Since super-enhancer marks (H3K27ac) have been demonstrated at the SOX1O gene in zebrafish and human melanoma cells (30), we tested whether arginine mutations could affect 
SKI repressor activity. For this, we expressed WT or R8 mutant SKI and examined (i) their recruitment to the SOX1O and PAX3 enhancers, (ii) HDAC activity, (iii) histone acetylation (H3K27ac), and (iv) cooccupancy of BRD4 (31-33). These studies revealed that, compared with WT SKI, R8 mutant SKI displayed enhanced recruitment at the SOX1O and PAX3 enhancers, increased HDAC, reduced $\mathrm{H} 3 \mathrm{~K} 27 \mathrm{ac}$, and reduced $\mathrm{BRD} 4$ recruitment (Figure 5J). These observations suggest that PRMT5 affects SOX10 and PAX3 expression by altering SKI repressor function.

SHARPIN-PRMT5 effect is important in SOX1O expression and melanoma growth in tumor with MTAP deletion. PRMT5 expression is upregulated in human melanoma tissues, and high expression is significantly associated with poorer overall survival $(P=0.00045$; Supplemental Figure 5, A and B). As mentioned, PRMT5 activity has been linked to the growth of tumors with deletion of MTAP (13, 34 ), leading to accumulation of its substrate MTA, which represses PRMT5 activity (13-15). As inhibition of the remaining PRMT5 activity in such tumors sensitizes them to cell death, we investigated the correlation between SHARPIN and PRMT5 activity in MTAP-deleted human melanomas.

Analysis of SHARPIN and SOX1O mRNA levels in a large cohort of human patient melanoma specimens (The Cancer Genome Atlas [https://cancergenome.nih.gov/], $n=472$ ) revealed that SHARPIN expression correlated strongly with $S O X 10$ expression $(r=0.572$, $P<0.0001, n=472$ ), but not with PAX3 expression (Figure 6, A and B). Interestingly, when we analyzed subsets of melanoma categorized as having low $(n=70)$ or high $(n=50)$ MTAP expression (Figure 6A), we found that SHARPIN expression was strongly correlated with both SOX1O and PAX3 levels in MTAP-low melanomas ( $r=0.458, P<0.0001 ; r=0.267, P=0.026$, respectively), but not in MTAP-high melanomas $(r=0.111, P=0.444 ; r=0.053, P=0.717$, respectively, Figure $6 \mathrm{~B})$. Likewise, a strong correlation between PRMT5 and PAX3 expression was found in the MTAP-high group $(r=0.576, P<0.0001$; Supplemental Figure 5C), but not in the MTAP-low group $(r=0.131, P=0.279)$. As PRMT5 levels did not correlate with $S O X 10$ expression in any of the melanoma groups, $S O X 10$ and $P A X 3$ expression may be differently regulated by MTAP (Supplemental Figure 5C). High SHARPIN expression was frequently observed in normal/low-MTAP melanomas, suggesting an inverse relationship between SHARPIN and MTAP expression and possibly PRMT5 activity (Supplemental Figure 5D).

Since subsets of human melanoma cells showed differential sensitivity to SHARPIN and PRMT5 KD (Figure 2, A and B), we further assessed the relationship among SHARPIN, PRMT5, and MTAP expression. In agreement with the in silico analyses of human melanoma specimens, we found that 3 cell lines (WM115, WM35, and UACC1389) that did not express MTAP exhibited low levels of SDME-RG and were sensitive to SHARPIN KD (Figure 2, A and $\mathrm{B}$, and Figure $6 \mathrm{C}$ ). Among the remaining SHARPIN KD-sensitive melanoma lines, 3 (WM793, Lu1205, and A375) exhibited reduced expression of SOX10 upon SHARPIN KD (Figure 2, A and B, Figure $3 \mathrm{C}$, and Figure 6C and Supplemental Figure 5E). SHARPIN-dependent SOX10 expression was detected in all SHARPIN KD-sensitive cells (Figure 2, A and B, Figure 3C and Supplemental Figure 5E). These observations suggest that MTAP- and SHARPIN-dependent SOX10 expression both define the susceptibility of melanoma cells to SHARPIN KD-induced growth inhibition (Figure 6C).
Coinhibition of SHARPIN and PRMT5 augments the melanoma growth inhibition. Ectopic expression of SHARPIN partially rescued the inhibition of PRMT5 activity (SDME-RG and activity assay) and melanoma growth by MTA (Figure 6D and Supplemental Figure 5F). Thus, while PRMT5 activity appears to be independently regulated by MTA and SHARPIN, SHARPIN can counteract the effect of MTA on PRMT5 activity. Indeed, preincubation of recombinant PRMT5/MEP50 complexes with purified SHARPIN protein increased the residual PRMT5 activity observed in the presence of MTA without changing percentage of inhibition of PRMT5 by MTA (Supplemental Figure 5G). In agreement, ectopic expression of MTAP in MTAP-deleted cells (WM35) increased PRMT5 activity (SDME-RG) and melanoma growth and partially rescued SHARPIN KD-induced growth inhibition in vitro and in vivo (Figure 7, A and B, and Supplemental Figure 5H), indicating that MTAP status influences the outcome of SHARPIN KD. Likewise, depletion of SHARPIN sensitized MTAP WT melanoma (from $12 \%$ to $30 \%$ inhibition) to PRMT5 inhibitor (Figure 7C), and double depletion of MTAP and SHARPIN caused a more pronounced growth inhibition compared with depletion of either gene alone (Figure 7, D and E).

Consistent with the in silico analysis of the TCGA data set (Figure 6, A and B), immunohistochemical staining of melanoma patient TMAs revealed a strong correlation between SOX10 and SHARPIN expression ( $r=0.586, P<0.0001, n=438)$, particularly in MTAP-low samples $(r=0.712, P<0.0001, n=59$; Figure 8, A and B, and Supplemental Figure 6). Moreover, high SOX10 expression correlated significantly with poor survival of patients in both the TCGA (Figure 8C) and TMA (Figure 8D) data sets. Interestingly, SHARPIN expression affected the survival only of patients with reduced MTAP levels (Figure 8, C and D), supporting the important role of SHARPIN in the survival of patients with MTAP-deleted melanomas.

Collectively, these results demonstrate that SHARPIN is a bona fide regulator of PRMT5 activity and that both proteins play a critical role in controlling melanoma growth through regulation of SOX10. Finally, SHARPIN-mediated PRMT5 regulation is associated with poor survival in patients with MTAP-deleted tumors.

\section{Discussion}

Deregulated protein methylation has been intimately linked to aberrant transcription of a number of tumor regulatory genes, including TP53. Here, we identified and characterized a new layer of arginine methylation regulation and demonstrated its implications for melanoma development. Our findings establish (i) the regulation of methyltransferase activity by a LUBAC adap-

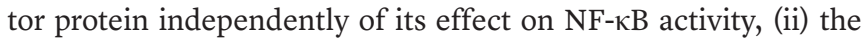
implications of SHARPIN-PRMT5 regulation for TGF- $\beta$ signaling via inactivation of SKI, (iii) the mechanism underlying regulation of SOX10 and PAX3, 2 key transcription factors in melanoma development, (iv) the significance of the SHARPIN-PRMT5 regulatory axis in tumors with MTAP deletion, and (v) the association between SHARPIN-PRMT5 regulation of SOX10-PAX3-MITF expression and therapy-resistant melanoma.

The effect of SHARPIN on PRMT5 is independent of HOIL-1L and HOIP and is thus unrelated to its known function in LUBAC. SHARPIN dimerization and its interaction with both the $\mathrm{N}$ and $\mathrm{C}$ 
termini of PRMT5 are expected to facilitate formation of active PRMT5 multiprotein complexes, which may underlie the SHARPIN-mediated increase in PRMT5 activation. The interaction of the SHARPIN UBL domain with PRMT5 (via the TIM barrel and $\beta$-barrel domains) and HOIP (via the UBA domain) raises interesting questions concerning the affinity of SHARPIN for the 3 complexes of which it is a component: LUBAC, PTEN, and PRMT5. Since ectopic expression of PRMT5 did not affect HOIP-SHARPIN interactions, we conclude that SHARPIN-PRMT5 binding does not disrupt the LUBAC complex. Nonetheless, the interaction between HOIP and PRMT5 has additional implications for crosstalk between LUBAC and PRMT5 complexes.

It is important to note that, while we found that SHARPIN KD or MTAP deletion reduced SDME-RG in nonhistone proteins, this deletion did not globally affect histone SDME-RG. This implies that global inhibition of histone methylation may require a more pronounced inhibition of PRMT5. Indeed, we have found that treatment with low doses of a PRMT5 inhibitor attenuated SDMERG of nonhistone proteins but not of H4R3me2s (our unpublished data). Thus, while SHARPIN does not affect arginine methylation of all histones, it does affect the expression of some gene networks, as demonstrated here for SOX1O and PAX3, through PRMT5-mediated methylation of R8 in the SKI repressor protein. These observations suggest that SHARPIN might regulate multiple aspects of PRMT5, including complex assembly (i.e., association with adaptor proteins such as Menin, pICln, RioK1), conformation, and posttranslational modification. In turn, these changes could influence the localization, substrate recognition and affinity, and/or activity of PRMT5 (35-37).

Our data favor the interpretation that SHARPIN modulates the activity, rather than the stability, of PRMT5. Among plausible mechanisms is SHARPIN-mediated recruitment of a ubiquitin ligase that mediates noncanonical ubiquitination of PRMT5. Since the effect of SHARPIN on PRMT5 in melanoma cells was only observed in cells with elevated SHARPIN levels, it is likely that this occurs independently of its LUBAC-associated function. Increased expression of SHARPIN has been reported in many other cancer types, suggesting that PRMT5 regulation might also be altered in those tumors. It is not yet known whether the SHARPIN-PRMT5 association is dependent on SHARPIN posttranslational modifications distinct from those required for its association with HOIL-1L and HOIP. In this context, it is possible that AKT, which is upregulated by SHARPIN-mediated suppression of PTEN, may contribute to its effects on PRMT5 activity, although this remains to be elucidated. We note that HIOL-1L or HOIP inhibition did not affect PRMT5 activity, melanoma growth, or sensitization to therapy, further supporting the independence of the LUBAC- and PRMT5-related functions of SHARPIN.

The ability of SHARPIN to increase MITF transcription by PRMT5-mediated inhibition of the SOX10 and PAX3 repressor SKI is a previously unknown mechanism of MITF regulation. Since MITF levels are crucial for determining melanoma development, metastasis, and therapy resistance, identifying the mechanisms that fine-tune the availability of SOX10 and PAX3 and, consequently, of MITF and pigmentation programs, has been of major interest. In addition to transcriptional regulation of SOX10 and PAX3 by SHARPIN, we observed an altered expres- sion of the SOX10/MITF protein by SHARPIN, independent of its transcript levels, pointing to an additional regulatory mechanism (Figure 2, A and B). Recently, transcriptomic analyses of PRMT5or MEP50-depleted lung cancer cells have suggested that these proteins regulate the TGF- $\beta$-mediated epithelial-mesenchymal transition through transcriptional regulation coupled to $\mathrm{H} 3$ and $\mathrm{H} 4$ arginine methylation (38). Along these lines, our results imply that PRMT5 regulation of PAX3 and SOX10 could involve transcriptional silencers other than SKI, such as SNO, which has also been implicated in TGF- $\beta$ signaling. SHARPIN-mediated control of TGF- $\beta$ signaling via PRMT5 regulation of SKI, which itself regulates TGF- $\beta$ signaling (39), thus identifies an additional regulatory axis with anticipated effects on the growth of melanoma as well as other tumor types.

SHARPIN-mediated activation of PRMT5 could override PRMT5 inhibition resulting from MTA accumulation in MTAPdeleted tumors. This suggests a molecular mechanism for fine-tuning PRMT5 activity and influencing the properties of such tumors. Suppression of the downstream PRMT5 targets PAX3 and MITF has been associated with therapy resistance, underscoring the advantages conferred by elevated SHARPIN expression and PRMT5 activity. Such tumors should be more sensitive to PRMT5 or SHARPIN inhibition, identifying a potential pathway for overcoming therapy resistance. Consistent with this, the melanoma cell lines defined in our study as sensitive to SHARPIN/PRMT5 KD express low levels of MTAP. Notably, longer survival of patients with MTAP-deleted tumors was correlated with lower SHARPIN expression levels. The roles of SHARPIN in NF- $\mathrm{BB}$ signaling, which is commonly upregulated in cancer, and in fine-tuning of PRMT5 activity therefore suggest that interventions that alter SHARPIN function may provide new potential therapeutic modalities, either alone or in combination with MTAP and/or PRMT5 inhibitors.

\section{Methods}

Animal studies. For xenograft experiments, WM115 or WM35 melanoma cell lines were modified for SHARPIN or MTAP expression with lentiviral vectors. Cells were injected subcutaneously (WM115, $4.0 \times$ $10^{6}$; WM35, $2.0 \times 10^{6}$ ) into the lower right flank of 6-week-old female nude mice (Envigo). Tumor sizes were monitored using calipers up to day 37 or 77 after injection.

Cell culture. HEK293T cells and the melanoma cell lines were cultured in DMEM medium (HyClone) containing 10\% FBS (Omega Scientific), $100 \mathrm{IU} / \mathrm{ml}$ penicillin, and $100 \mu \mathrm{g} / \mathrm{ml}$ streptomycin. All UACC melanoma cell lines were maintained in RPMI 1640 medium (Corning) supplemented with 10\% FBS and antibiotics as above. Cells were maintained in growth phase and did not exceed $80 \%$ confluency. Melanoma cell lines were obtained from Wistar Institute (WM series), TGen (UACC series), National Cancer Institute (NCI), and ATCC. For cell line authentication, short tandem repeat (STR) analysis was performed on isolated genomic DNA with the GenePrint 10 System (Promega), and peaks were analyzed using GeneMarker HID from Softgenetics. Allele calls were searched against STR databases maintained by ATCC (www.atcc.org), DSMZ (www.dsmz.de), Texas Tech University Children's Oncology Group (cogcell.org), and the Wistar Institute Melanoma Cell STR Profiles (http://www.wistar.org/ lab/meenhard-herlyn-dvm-dsc/page/melanoma-cell-str-profiles). Authentication was last performed on August 24, 2015. 
Antibodies and reagents. Antibodies to the following proteins were purchased or obtained as indicated: I $\mathrm{I} B \alpha$ (L35A5), p-p65 (93H1), p65 (L8F6), H3K27me3 (C36B11), H3K27ac (D5E4), BRD4 (E2A7X), lamin A/C (4C11), CREB (86B10), p-CREB (87G3), p-ATF2 (catalog 9225), SDME-RG (catalog 13222), MEP50 (catalog 2823), SOX10 (D5V9L), PAX3 (catalog 12412), and ATF2 (20F1, Cell Signaling Technology), FLAG (F3165, Sigma-Aldrich), MITF (C5) and H4R3me2s (ab5823, Abcam), PRMT5 (730054), V5 (E10/V4RR, Life Technologies), tubulin (D-10), SOX10 (N-20), PAX3 (N-19), MEP50 (FG-4), PRMT5 (A-11), GAPDH (6C5), $\beta$-actin (AC-15), p53 (DO-7), Smad2/3 (FL-425), HDAC3 (H-99), SKI (H-329), Myc (4E10, Santa Cruz Biotechnology Inc.), c-SKI (A303-518, Bethyl Laboratory), HOIP, HOIL-1L, SHARPIN (gift from the Iwai laboratory), MTAP (11475-1-AP, Proteintech), and SYM10 (07-412, Millipore). MTA was from Millipore.

Recombinant proteins. Recombinant human TGF- $\beta$ was purchased from R\&D Systems, and recombinant human PRMT5/ MEP50 was from Sigma-Aldrich. Human SHARPIN (full length and $\triangle \mathrm{UBL}$ mutant) proteins were produced using pET15b plasmid (Novagen) transformed into BL21(DE3)pLysS competent cells (Agilent). Cultured bacteria were incubated with lysis buffer $(50 \mathrm{mM}$ $\mathrm{NaH}_{2} \mathrm{PO}_{4}, 300 \mathrm{mM} \mathrm{NaCl}, 20 \mathrm{mM}$ 2-mercaptoethanol, $5 \mathrm{mM}$ imidazole, adjusted to $\mathrm{pH} 8.0$ ) supplemented with $1 \times$ protease inhibitor cocktail (Roche) and lysozyme $(1 \mathrm{mg} / \mathrm{ml})$ for 30 minutes on ice. The samples were sonicated to reduce viscosity and centrifuged, and the supernatant was collected and incubated with HisPur Ni-NTA Resin (Thermo Scientific) for 3 hours. Ni-NTA beads were washed with lysis buffer containing $10 \mathrm{mM}$ imidazole and then incubated with elution buffer (200 mM imidazole) with gentle shaking at $4^{\circ} \mathrm{C}$ for 1 hour. The purified protein was dialyzed overnight in PBS using a Slide-A-Lyzer cassette (Pierce).

Immunoblot analysis and gel filtration. Cells were lysed by incubation in TBS-lysis buffer (consisting of TBS [50 mM Tris- $\mathrm{HCl}, \mathrm{pH}$ $7.5,150 \mathrm{mM} \mathrm{NaCl}$ s supplemented with $1 \%$ [v/v] Triton X-100 and $1 \times$ protease inhibitor cocktail, Roche) for 20 minutes at $4^{\circ} \mathrm{C}$. For immunoprecipitation, lysates were incubated overnight at $4^{\circ} \mathrm{C}$ with the appropriate antibodies and then with protein $\mathrm{A} / \mathrm{G}$ agarose beads (Santa Cruz Biotechnology Inc.). Proteins were eluted by the addition of lysis buffer and boiled in Laemmli buffer before separation by SDSPAGE and transfer to a PVDF membrane. Membranes were incubated for 1 hour at room temperature with blocking solution (TBS containing $0.1 \%$ Tween 20 and $5 \%$ nonfat milk) followed by incubation overnight at $4^{\circ} \mathrm{C}$ with the appropriate primary antibody. Membranes were washed with TBS and then incubated for 1 hour at room temperature with secondary antibody (Alexa Fluor 680-conjugated goat anti-rabbit, goat anti-mouse, or donkey anti-goat [Life Technologies] or IRDye 800-conjugated goat anti-mouse [Rockland Immunochemicals]). Protein bands were visualized and quantified using an Odyssey Infrared Imaging System (LiCor Biosciences).

For gel filtration, WM793 and WM115 cells were lysed in lysis buffer (50 mM Tris, $\mathrm{pH} 7.5,1 \mathrm{mM} \mathrm{MgCl}, 1 \mathrm{mM}$ dithiothreit$\mathrm{ol}$, and protease inhibitor cocktail) by repeatedly passing through a 27-gauge, 1/2-inch long needle. The cell lysates (3 mg, WM793; $1.5 \mathrm{mg}$, WM115) were cleared by centrifugation at 100,000 $\mathrm{g}$ for 60 minutes and loaded onto a Superdex 200 column operated by a Pharmacia/GE ÄKTA FPLC (buffer: 50 mM Tris-HCl, pH 7.5, 150 $\mathrm{mM} \mathrm{NaCl}, 1 \mathrm{mM}$ EDTA). Fractions $(0.5 \mathrm{ml})$ were collected and analyzed by SDS-PAGE and immunoblotting.
Luciferase assay. Cultures were transfected with the TGF- $\beta$ reporter plasmid (CAGA)9-Luc and the control plasmid p-CMV-Cypridina and lysed 8 or 24 hours later. (CAGA) 9 luciferase activity was measured using a Luciferase Assay system (Promega) with a Veritas microplate luminometer (Promega). Cypridina luciferase activity was measured with a Luciferase Flash Assay Kit (Thermo Scientific).

Gene silencing. Gene-specific shRNA lentiviral vectors with a pLKO.1 backbone were obtained from the La Jolla Institute for Allergy and Immunology RNAi Center (La Jolla, California, USA). Lentiviral particles were prepared using standard protocols. Briefly, shRNA plasmid and the second generation of packaging plasmids $\Delta R 8.2$ and Vsv-G (Addgene) were transfected into HEK293T cells. Viral supernatants were collected after 48 hours of culture and used with polybrene (Sigma-Aldrich) for infection of melanoma cell lines. For SKI RNAi, cells were transfected with 250 pmol of siRNA against SKI or the corresponding scrambled siRNA (SMARTpool reagents, Dharmacon) using jetPRIME (Polyplus-transfection).

DNA constructs and transfection. DNA plasmids were transfected into the indicated cell lines using jetPrime, except for WM115, which was electroporated with an Amaxa Nucleofector II (Lonza) according to the manufacturer's protocol. pLX304-SOX10, pLX304-PRMT5, pLX304-MEP50, and $\mathrm{pLX304-MTAP}$ lentiviral overexpression vectors were obtained from DNASU (Arizona State University Biodesign Institute, Tempe, Arizona, USA). pcDNA3.1-FLAG-SHARPIN and pcDNA3.2-Myc-SHARPIN were generated by the Iwai laboratory, and pcDNA3-FLAG-SKI was a kind gift from the Miyazawa Laboratory (University of Yamanashi, Köfu, Japan). The SHARPIN ORF was inserted into pLENTI-puro (Addgene, 39481) using BamHI and EcoRI sites to generate the lentiviral SHARPIN overexpression plasmid. pcDNA3.1-PRMT5-WT and mutant plasmids were produced from pLX304-PRMT5. pcDNA3-FLAG-SKI R8K and R658/660K mutant plasmids were generated using the QuikChange II XL SiteDirected Mutagenesis Kit (Agilent).

MS. WM115 cells were transfected with pcDNA3.1-Flag-SHARPIN or the control pcDNA3.1-Flag (empty vector) plasmids and lysed in TBS-lysis buffer (described above). Lysates were precleared with protein $\mathrm{A} / \mathrm{G}$ beads (Santa Cruz Biotechnology Inc.) for 1 hour at $4^{\circ} \mathrm{C}$ and immunoprecipitated with FLAG-M2-agarose beads (SigmaAldrich) overnight at $4^{\circ} \mathrm{C}$. Beads were washed with TBS-lysis buffer and TBS containing $1 \times$ protease inhibitor cocktail and then subjected to on-bead tryptic digestion followed by MS (40).

RNA extraction and quantitative PCR. Total RNA was isolated from cells with GenElute (Sigma-Aldrich) and reverse transcribed using high-capacity cDNA synthesis kits (Applied Biosystems). Quantitative PCR (qPCR) was performed with CFX Connect Real-Time PCR Detection System (Bio-Rad) using FastStart Universal SYBR Green Master Mix (Life Technologies). GAPDH was amplified as a control. Sequence-specific primers are shown in Supplemental Table 2.

ChIP. Cells were fixed in 1\% formaldehyde in PBS for 10 minutes at room temperature and then incubated with $0.125 \mathrm{M}$ glycine for 5 minutes. The cells were washed with PBS, incubated in lysis buffer (50 mM Tris-HCl, pH 8.0, 1\% SDS, 10 mM EDTA), and sonicated on ice to shear the DNA into approximately 500-bp fragments. The sonicate was centrifuged, and the supernatant was precleared by incubation with protein A/G beads (Santa Cruz Biotechnology Inc.) on a rotating platform at $4^{\circ} \mathrm{C}$ for 1 hour. Control IgG or the appropriate primary antibody was added to the sample and incubated overnight at $4^{\circ} \mathrm{C}$. Protein $\mathrm{A} / \mathrm{G}$ beads were added, and the 
mixture was incubated at $4^{\circ} \mathrm{C}$ for 2 hours. The beads were washed sequentially with low-salt buffer (20 mM Tris- $\mathrm{HCl}, \mathrm{pH}$ 8.0, 0.1\% SDS, 1\% Triton X-100, 2 mM EDTA, $150 \mathrm{mM} \mathrm{NaCl}$ ), high-salt buffer (as for low salt except $500 \mathrm{mM} \mathrm{NaCl}$ ), and $\mathrm{LiCl}$ wash buffer (10 $\mathrm{mM}$ Tris-HCl, pH 8.0, $0.25 \mathrm{M} \mathrm{LiCl,1 \%} \mathrm{NP}-40,1 \%$ sodium deoxycholate, $1 \mathrm{mM}$ EDTA). Chromatin was eluted in $120 \mu$ l elution buffer $\left(1 \% \mathrm{SDS}, 100 \mathrm{mM} \mathrm{NaHCO}{ }_{3}\right.$ ) for 15 minutes at $30^{\circ} \mathrm{C}$. Samples were centrifuged, and the supernatant was mixed with $4.8 \mu \mathrm{l}$ of $5 \mathrm{M} \mathrm{NaCl}$ and incubated overnight at $65^{\circ} \mathrm{C}$. RNase A $(2 \mu \mathrm{lof} 10 \mathrm{mg} / \mathrm{ml})$ and proteinase $\mathrm{K}(2 \mu \mathrm{l}$ of $20 \mathrm{mg} / \mathrm{ml})$ were added, and the sample was incubated with gentle shaking at $45^{\circ} \mathrm{C}$ for 1 hour and then extracted with phenol/chloroform to isolate the DNA. The samples were subjected to qPCR to detect the promoter region of the target gene using the primers shown in Supplemental Table 2.

Blue native-PAGE. Cells were incubated in lysis buffer $(20 \mathrm{mM}$ Bis-tris, pH 7.0, $500 \mathrm{mM} \varepsilon$-aminocaproic acid, $20 \mathrm{mM} \mathrm{NaCl}, 0.2 \mathrm{mM}$ EDTA, 0.5\% Triton X-100, 10\% glycerol, and protease inhibitor cocktail) and then dialyzed against lysis buffer containing $0.1 \%$ Triton X-100 overnight (41). Samples were loaded onto a $4 \%-15 \%$ gradient gel and resolved for 3.5 hours at $150 \mathrm{~V}$. The gel was transferred to a PVDF membrane (30 V for 8 hours) using Tris-glycine buffer ( $\mathrm{pH} 8.3$ ). The membrane was decolorized in 100\% methanol, blocked with 5\% nonfat milk, and then incubated with the appropriate primary antibody overnight followed by horseradish peroxidase-conjugated secondary antibody (Cell Signaling Technology). Signals were developed using Western Lightning Plus-ECL (PerkinElmer).

For 2D SDS-PAGE, the Blue Native-PAGE gel was sliced, incubated in $2 \times$ SDS sample buffer for 10 minutes at room temperature, and microwaved for 20 seconds. Treated gel slices were loaded onto 4\%-20\% gradient SDS-PAGE gels, resolved, and transferred to membranes under standard conditions.

Cell viability/cell death assays. Cell growth was assayed using ATPlite (PerkinElmer) according to the manufacturer's instructions. WM793 melanoma cells were seeded at $5 \times 10^{3}$ cells/well in 96-well plates and incubated with etoposide $(5 \mu \mathrm{M})$ or vehicle for 72 hours. Cells were then incubated with ATPlite reagents, and viability was quantified by monitoring luminescence intensity with FlexStation 3 (Molecular Devices). For flow cytometric live/dead cell discrimination, cells were stained with annexin V (Alexa Fluor 488) and propidium iodide (PI) according to the manufacturer's protocol (Life Technologies) and analyzed on a FACSCanto flow cytometer.

Colony-forming efficiency. Cells were seeded in 6-well plates at $10^{3} /$ well and cultured for 2 weeks. The colonies were stained with crystal violet (PBS, pH 7.4, 0.2\% [w/v] crystal violet, $4 \%$ [w/v] paraformaldehyde) and visualized. Colonies were enumerated using ImageJ software (NIH).

Tumor microarray analysis. Melanoma TMA samples were obtained from Yale University and stained by incubation with primary antibodies to MTAP (1:50 dilution, Proteintech), SOX10 (1:50 dilution, Santa Cruz Biotechnology Inc.), and SHARPIN (1:25 dilution, Sigma-Aldrich) and then with fluorescein-conjugated secondary antibodies. Slides were counterstained with DAPI and the images were scanned using an Aperio ScanScope. The relative staining intensity was assessed in a blinded fashion by 2 independent investigators.

Immunocytochemistry. Cells were cultured on glass coverslips, fixed in $4 \%$ paraformaldehyde for 10 minutes, washed in PBS, permeabilized with $0.25 \%$ Triton X-100 in PBS ( $\mathrm{pH} 7.4$ ) for 20 min- utes, and blocked in 3\% BSA (w/v) in PBS at room temperature for 1 hour. Cells were incubated with the appropriate primary antibody for 16 hours at $4^{\circ} \mathrm{C}$ and then with the corresponding Alexa Fluor 568-conjugated secondary antibody (Life Technologies) for 1 hour at room temperature.

Subcellular fractionation. Cell lysates were prepared by incubation in hypotonic buffer (20 mM HEPES, pH 7.4, $250 \mathrm{mM}$ sucrose, $0.2 \%$ Triton X-100, $10 \mathrm{mM} \mathrm{KCl}, 1.5 \mathrm{mM} \mathrm{MgCl}$, 1 mM EDTA, $1 \mathrm{mM}$ EGTA, and protease inhibitor cocktail). The lysate was centrifuged for 10 minutes at $800 \mathrm{~g}$, and the supernatant was designated the cytosolic fraction. The cell pellet was resuspended in RIPA buffer $(10 \mathrm{mM}$ Tris-HCl, pH 7.5, 1 mM EDTA, 0.5 mM EGTA, 1\% Triton X-100, 0.5\% deoxycholate, $0.1 \%$ SDS, $150 \mathrm{mM} \mathrm{NaCl}$, and $1 \times$ protease inhibitor cocktail), incubated for 20 minutes on ice, and sonicated (10 seconds, $20 \%$ amplitude) using a microtip (Misonix XL2020). The sample was centrifuged for 20 minutes at $16,100 \mathrm{~g}$, and the supernatant was designated the nuclear fraction.

In vitro methyltransferase assay and PRMT5 activity assay. The enzymatic activity of PRMT5 (recombinant protein, nuclear extracts, or anti-PRMT5 immunoprecipitates) was measured using an Epigenase PRMT Methyltransferase (Type II-Specific) Activity/Inhibition Assay Kit (Epigentek) according to the the manufacturer's protocol. The assay kit contained histone 4 peptide as a substrate and an SDME-RG-specific antibody for detection.

In vitro methylation of histone 4 protein was performed as described previously (42) with slight modifications. Histone 4 protein $(3 \mu \mathrm{g})$ was incubated with $200 \mathrm{ng}$ of PRMT5/MEP50, $250 \mathrm{ng}$ of SHARPIN full-length or $500 \mathrm{ng} \Delta \mathrm{UBL}$, and $2 \mu \mathrm{Ci}$ of $S$-adenosyl-L-methyl- ${ }^{3} \mathrm{H}$-methionine $\left({ }^{3} \mathrm{H}-\mathrm{SAM}\right.$, PerkinElmer) in $20 \mu \mathrm{l}$ of reaction mixture with HMTase buffer ( $25 \mathrm{mM} \mathrm{NaCl}, 25 \mathrm{mM}$ Tris, $\mathrm{pH} 8.8$ ) for 90 minutes at $30^{\circ} \mathrm{C}$. The reactions were resolved on $4 \%-20 \%$ gradient SDS-PAGE gels and transferred to PVDF membranes. The membranes were treated with enhancing spray (PerkinElmer), air-dried, and subjected to autoradiography.

Statistics. All data are expressed as mean \pm SD unless specified. Group differences were analyzed using Student's $t$ test (unpaired, 2 tailed). Statistical analyses of multiple groups within the experiment were performed using 1- or 2-way ANOVA with appropriate multicomparison correction (Dunnett's, Tukey's test). All analyses were performed using Prism 7.0 software (GraphPad). TCGA and gene expression data are expressed as median \pm interquartile range. Differences were analyzed using Mann-Whitney $U$ test ( 2 tailed) with Prism 7.0 software. Xenograft data were analyzed using 2-way ANOVA for tumor volume analysis and Welch's test (unpaired, 2-tailed Student $t$ test with Welch's correction). $P$ values $\leq 0.05$ were considered statistically significant. For patient survival analysis, data were analyzed using log-rank test with Prism 7.0 software.

Study approval. Animal studies were approved (AUF 15-089 with amendment 6649) by the Institutional Animal Care and Use Committee of Sanford Burnham Prebys Medical Discovery Institute. Animal care followed institutional guidelines.

\section{Author contributions}

Hyungsoo Kim and ZAR designed the experiments. HT, Heejung Kim, OK, YF, JYH, Hyungsoo Kim, and SJS conducted the experiments. H. Kluger, LJ, TZ, KB, and HZ analyzed the data. KI and AM provided reagents. Hyungsoo Kim and ZAR wrote the manuscript. 


\section{Acknowledgments}

We thank Keiji Miyazawa (University of Yamanashi) for providing expression vectors, and members of the Ronai lab for discussions. This work was supported by grants from the NCI (R21CA198468 to HK; R35CA197465 to ZAR) and the Shantou University-Technion Research Program (to ZAR).
Address correspondence to: Ze'ev A. Ronai or Hyungsoo Kim, 10901 North Torrey Pines Road, La Jolla, California, 92037, USA. Phone: 858.646.3185; Email: zeev@ronailab.net (Z.A. Ronai) or hkim@sbpdiscovery.org (H. Kim). Or to: Ze'ev A. Ronai, Technion Integrated Cancer Center, Faculty of Medicine, Technion, Haifa, Israel, 31096. Phone: 04.829.5447; Email: ronai@technion.ac.il.
1. Stieglitz B, et al. Structural basis for ligase-specific conjugation of linear ubiquitin chains by HOIP. Nature. 2013;503(7476):422-426.

2. Ikeda F, et al. SHARPIN forms a linear ubiquitin ligase complex regulating NF- $\kappa \mathrm{B}$ activity and apoptosis. Nature. 2011;471(7340):637-641.

3. Tokunaga $\mathrm{F}$, et al. SHARPIN is a component of the $\mathrm{NF}-\kappa \mathrm{B}$-activating linear ubiquitin chain assembly complex. Nature. 2011;471(7340):633-636.

4. Yang Y, et al. Essential role of the linear ubiquitin chain assembly complex in lymphoma revealed by rare germline polymorphisms. Cancer Discov. 2014;4(4):480-493

5. MacKay C, et al. E3 ubiquitin ligase HOIP attenuates apoptotic cell death induced by cisplatin. Cancer Res. 2014;74(8):2246-2257.

6. Damgaard RB, et al. The deubiquitinase OTULIN is an essential negative regulator of inflammation and autoimmunity. Cell.2016;166(5):1215-1230.e20.

7. He L, Ingram A, Rybak AP, Tang D. Shank-interacting protein-like 1 promotes tumorigenesis via PTEN inhibition in human tumor cells. J Clin Invest. 2010;120(6):2094-2108.

8. Li J, et al. SHARPIN overexpression induces tumorigenesis in human prostate cancer $\mathrm{LNCaP}$, DU145 and PC-3 cells via NF- $\kappa \mathrm{B} / \mathrm{ERK} /$ Akt signaling pathway. Med Oncol. 2015;32(2):444

9. Bii VM, Rae DT, Trobridge GD. A novel gammaretroviral shuttle vector insertional mutagenesis screen identifies SHARPIN as a breast cancer metastasis gene and prognostic biomarker. Oncotarget. 2015;6(37):39507-39520.

10. Stopa N, Krebs JE, Shechter D. The PRMT5 arginine methyltransferase: many roles in development, cancer and beyond. Cell Mol Life Sci. 2015;72(11):2041-2059.

11. Jansson M, et al. Arginine methylation regulates the 553 response. Nat Cell Biol. 2008;10(12):1431-1439.

12. Zhang B, et al. Targeting protein arginine methyltransferase 5 inhibits colorectal cancer growth by decreasing arginine methylation of eIF4E and FGFR3. Oncotarget. 2015;6(26):22799-22811.

13. Kryukov GV, et al. MTAP deletion confers enhanced dependency on the PRMT5 arginine methyltransferase in cancer cells. Science. 2016;351(6278):1214-1218.

14. Mavrakis KJ, et al. Disordered methionine metabolism in MTAP/CDKN2A-deleted cancers leads to dependence on PRMT5. Science. 2016;351(6278):1208-1213.

15. Marjon K, et al. MTAP deletions in cancer create vulnerability to targeting of the MAT2A/PRMT5/ RIOK1 axis. Cell Rep. 2016;15(3):574-587.

16. Shah $\mathrm{M}$, et al. A role for ATF2 in regulating MITF and melanoma development. PLoS Genet. 2010;6(12):e1001258.

17. Pierrat MJ, Marsaud V, Mauviel A, Javelaud D. Expression of microphthalmia-associated transcription factor (MITF), which is critical for melanoma progression, is inhibited by both transcription factor GLI2 and transforming growth factor- $\beta$. J Biol Chem. 2012;287(22):17996-18004.

18. Carreira S, et al. Mitf regulation of Dia1 controls melanoma proliferation and invasiveness. Genes Dev. 2006;20(24):3426-3439.

19. Yagi S, Hirosawa M, Shiota K. DNA methylation profile: a composer-, conductor-, and player-orchestrated Mammalian genome consisting of genes and transposable genetic elements. J Reprod Dev. 2012;58(3):265-273.

20. Burgos ES, et al. Histone $\mathrm{H} 2 \mathrm{~A}$ and $\mathrm{H} 4 \mathrm{~N}$-terminal tails are positioned by the MEP50 WD repeat protein for efficient methylation by the PRMT5 arginine methyltransferase. J Biol Chem. 2015;290(15):9674-9689.

21. Antonysamy S, et al. Crystal structure of the human PRMT5:MEP50 complex. Proc Natl Acad Sci U S A. 2012;109(44):17960-17965.

22. Nicholas C, et al. PRMT5 is upregulated in malignant and metastatic melanoma and regulates expression of MITF and p27(Kip1.). PLoS One. 2013;8(9):e74710.

23. Pal S, Vishwanath SN, Erdjument-Bromage H, Tempst P, Sif S. Human SWI/SNF-associated PRMT5 methylates histone $\mathrm{H} 3$ arginine 8 and negatively regulates expression of ST7 and NM23 tumor suppressor genes. Mol Cell Biol. 2004;24(21):9630-9645.

24. Wang L, Pal S, Sif S. Protein arginine methyltransferase 5 suppresses the transcription of the RB family of tumor suppressors in leukemia and lymphoma cells. Mol Cell Biol. 2008;28(20):6262-6277.

25. Yang $\mathrm{G}$, et al. Inhibition of PAX3 by TGF-beta modulates melanocyte viability. Mol Cell. 2008;32(4):554-563.

26. John N, Cinelli P, Wegner M, Sommer L. Transforming growth factor $\beta$-mediated Sox 10 suppression controls mesenchymal progenitor generation in neural crest stem cells. Stem Cells. 2011;29(4):689-699.

27. Tabata T, Kokura K, Ten Dijke P, Ishii S. Ski corepressor complexes maintain the basal repressed state of the TGF-beta target gene, SMAD7, via HDAC3 and PRMT5. Genes Cells. 2009;14(1):17-28.

28. Nagata M, et al. Nuclear and cytoplasmic c-Ski differently modulate cellular functions. Genes Cells. 2006;11(11):1267-1280.

29. Thandapani P, O'Connor TR, Bailey TL, Richard S. Defining the RGG/RG motif. Mol Cell. 2013;50(5):613-623.
30. Kaufman CK, et al. A zebrafish melanoma model reveals emergence of neural crest identity during melanoma initiation. Science. 2016;351(6272):aad2197.

31. Nomura T, et al. Ski is a component of the histone deacetylase complex required for transcriptional repression by Mad and thyroid hormone receptor. Genes Dev. 1999;13(4):412-423.

32. Ueki N, Zhang L, Hayman MJ, Haymann MJ. Ski can negatively regulates macrophage differentiation through its interaction with PU.1. Oncogene. 2008;27(3):300-307.

33. Zippo A, Serafini R, Rocchigiani M, Pennacchini S, Krepelova A, Oliviero S. Histone crosstalk between H3S10ph and H4K16ac generates a histone code that mediates transcription elongation. Cell. 2009;138(6):1122-1136.

34. Christopher SA, Diegelman P, Porter CW, Kruger WD. Methylthioadenosine phosphorylase, a gene frequently codeleted with p16(cdkN2a/ARF), acts as a tumor suppressor in a breast cancer cell line. Cancer Res. 2002;62(22):6639-6644.

35. Gurung B, Hua X. Menin/PRMT5/hedgehog signaling: a potential target for the treatment of multiple endocrine neoplasia type 1 tumors. Epigenomics. 2013;5(5):469-471.

36. Meister G, Eggert C, Bühler D, Brahms H, Kambach C, Fischer U. Methylation of Sm proteins by a complex containing PRMT5 and the putative U snRNP assembly factor pICln. Curr Biol. 2001;11(24):1990-1994.

37. Guderian G, et al. RioK1, a new interactor of protein arginine methyltransferase 5 (PRMT5), competes with pICln for binding and modulates PRMT5 complex composition and substrate specificity. J Biol Chem. 2011;286(3):1976-1986.

38. Chen H, Lorton B, Gupta V, Shechter D. A TGF $\beta$-PRMT5-MEP50 axis regulates cancer cel invasion through histone $\mathrm{H} 3$ and $\mathrm{H} 4$ arginine methylation coupled transcriptional activation and repression. Oncogene. 2017;36(3):373-386.

39. Luo K, et al. The Ski oncoprotein interacts with the Smad proteins to repress TGFbeta signaling. Genes Dev. 1999;13(17):2196-2206.

40. Brill LM, Motamedchaboki K, Wu S, Wolf DA. Comprehensive proteomic analysis of Schizosaccharomyces pombe by two-dimensional HPLC-tandem mass spectrometry. Methods. 2009;48(3):311-319.

41. Fiala GJ, Schamel WW, Blumenthal B. Blue native polyacrylamide gel electrophoresis (BN-PAGE) for analysis of multiprotein complexes from cellular lysates. J Vis Exp. 2011;(48):2164.

42. Rea S, et al. Regulation of chromatin structure by site-specific histone $\mathrm{H} 3$ methyltransferases. Nature. 2000;406(6796):593-599. 